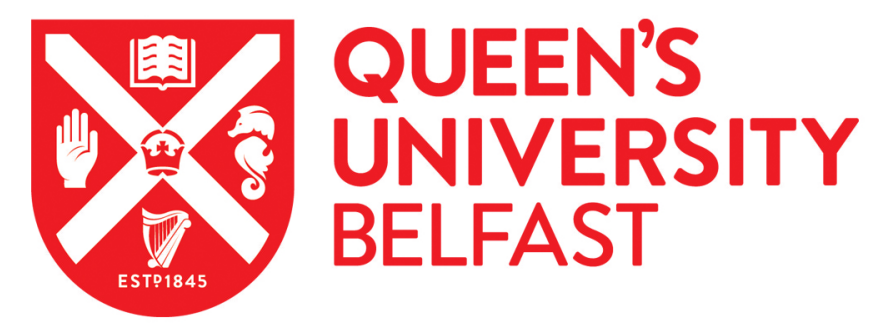

\title{
Properties of the Bare Nucleus of Comet 96P/Machholz 1
}

\author{
Eisner, N. L., Knight, M. M., Snodgrass, C., Kelley, M. S. P., Fitzsimmons, A., \& Kokotanekova, R. (2019). \\ Properties of the Bare Nucleus of Comet 96P/Machholz 1. Astronomical Journal, 157(5), [186]. \\ https://doi.org/10.3847/1538-3881/ab0f42
}

Published in:

Astronomical Journal

Document Version:

Publisher's PDF, also known as Version of record

Queen's University Belfast - Research Portal:

Link to publication record in Queen's University Belfast Research Portal

Publisher rights

(c) 2019 The American Astronomical Society. All rights reserved.

This work is made available online in accordance with the publisher's policies. Please refer to any applicable terms of use of the publisher.

\section{General rights}

Copyright for the publications made accessible via the Queen's University Belfast Research Portal is retained by the author(s) and / or other copyright owners and it is a condition of accessing these publications that users recognise and abide by the legal requirements associated with these rights.

Take down policy

The Research Portal is Queen's institutional repository that provides access to Queen's research output. Every effort has been made to ensure that content in the Research Portal does not infringe any person's rights, or applicable UK laws. If you discover content in the Research Portal that you believe breaches copyright or violates any law, please contact openaccess@qub.ac.uk. 


\title{
Properties of the Bare Nucleus of Comet 96P/Machholz $1^{*}$
}

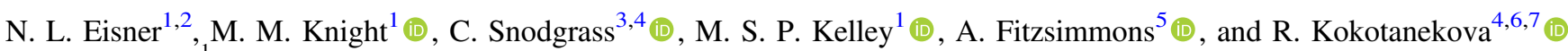 \\ ${ }^{1}$ Department of Astronomy, University of Maryland, College Park, MD 20742, USA; nora.eisner@physics.ox.ac.uk \\ ${ }^{2}$ Department of Astrophysics, University of Oxford, Oxford, UK \\ ${ }^{3}$ Institute for Astronomy, University of Edinburgh, Royal Observatory, Edinburgh EH9 3HJ, UK \\ ${ }^{4}$ Planetary and Space Sciences, School of Physical Sciences, The Open University, Milton Keynes, MK7 6AA, UK \\ 5 Astrophysics Research Centre, Queen's University Belfast, Belfast BT7 1NN, UK \\ ${ }^{6}$ European Southern Observatory, Karl-Schwarzschild-Strasse 2, D-85748 Garching bei München, Germany \\ ${ }^{7}$ Max-Planck-Institut für Sonnensystemforschung, Justus-von-Liebig-Weg 3, D-37077 Göttingen, Germany \\ Received 2018 December 7; revised 2019 March 7; accepted 2019 March 9; published 2019 April 22
}

\begin{abstract}
We observed comet 96P/Machholz 1 on a total of nine nights before and after perihelion during its 2017/2018 apparition. Both its unusually small perihelion distance and the observed fragmentation during multiple apparitions make 96P an object of great interest. Our observations show no evidence of a detectable dust coma, implying that we are observing a bare nucleus at distances ranging from 2.3 to $3.8 \mathrm{au}$. Based on this assumption, we calculated its color and found average values of $g^{\prime}-r^{\prime}=0.50 \pm 0.04, r^{\prime}-i^{\prime}=0.17 \pm 0.03$, and $i^{\prime}-z^{\prime}=0.06 \pm 0.04$. These are notably more blue than those of the nuclei of other Jupiter-family and long-period comets. Furthermore, assuming a bare nucleus, we found an equivalent nuclear radius of $3.4 \pm 0.2 \mathrm{~km}$ with an axial ratio of at least $1.6 \pm 0.1$. The lightcurve clearly displays one large peak, one broad flat peak, and two distinct troughs, with a clear asymmetry that suggests that the shape of the nucleus deviates from that of a simple triaxial ellipsoid. This asymmetry in the lightcurve allowed us to constrain the nuclear rotation period to $4.10 \pm 0.03 \mathrm{hr}$ and $4.096 \pm 0.002 \mathrm{hr}$ before and after perihelion, respectively. Within the uncertainties, 96P's rotation period does not appear to have changed throughout the apparition, and we conclude a maximum possible change in rotation period of $130 \mathrm{~s}$. The observed properties were compared to those of comet 322P and interstellar object 1I/'Oumuamua in an attempt to study the effects of close perihelion passages on cometary surfaces and their internal structure and the potential interstellar origin of $96 \mathrm{P}$.
\end{abstract}

Key words: comets: individual (96P/Machholz 1) - methods: data analysis - methods: observational

Supporting material: machine-readable table

\section{Introduction}

In this paper, we focus on analyzing the properties of the unusual comet 96P/Machholz 1 (henceforth 96P). Although 96P has a short orbital period ( $\sim 5.3 \mathrm{yr})$, its orbit is highly inclined $\left(\sim 58^{\circ}\right)$ and it has a very low perihelion distance $(q$; currently $0.124 \mathrm{au}$ ). These properties are very different from typical Jupiter-family comet orbits. $96 \mathrm{P}$ has been shown to be trapped in a Kozai resonance with Jupiter (e.g., Levison \& Dones 2014), where its eccentricity and inclination oscillate out of phase but with the same frequency. Orbital integrations (e.g., Green et al. 1990; McIntosh 1990) find that it oscillates from a high-inclination orbit (near $80^{\circ}$ ) with perihelion near 1 au, to a low-inclination orbit with perihelion at $\sim 0.03 \mathrm{au}$, and back again on a timescale of $\sim 4000 \mathrm{yr}$. Although 96P's Tisserand parameter $\left(T_{J}\right)$ is currently 1.94 , Bailey et al. (1992) showed that $T_{J}$ was greater than 2 a few thousand years ago and has been slowly drifting downward. Given that $T_{J}=2.0$ is the canonical divider (e.g., Carusi et al. 1987) between

\footnotetext{
* Based on observations obtained at the Southern Astrophysical Research (SOAR) telescope, which is a joint project of the Ministério da Ciência, Tecnologia, Inovações e Comunicações do Brasil (MCTIC/LNA), the U.S. National Optical Astronomy Observatory (NOAO), the University of North Carolina at Chapel Hill (UNC), and Michigan State University (MSU). Also based on service observations made with the William Herschel Telescope operated on the island of La Palma by the Isaac Newton Group of Telescopes in the Spanish Observatorio del Roque de los Muchachos of the Instituto de Astrofísica de Canarias. Also based on observations collected at the European Southern Observatory under ESO program 0101.C-0709(A).
}

Jupiter-family orbits $\left(2.0<T_{J}<3.0\right)$ and Halley-type or Oort cloud orbits $\left(T_{J}<2.0\right)$, 96P's origin is uncertain.

Comet 96P is generally difficult to study: it has a small solar elongation when near perihelion, it never comes particularly close to Earth because of its high inclination, and its high southern declination throughout most of its orbit limits the telescopes capable of observing it. It has been observed on every apparition since 1996 by the space-based SOlar and Heliospheric Observatory (SOHO)'s SWAN UV imager (Combi et al. 2011, 2019) and LASCO optical imager (Grynko et al. 2004; Eisner et al. 2018). However, ground-based studies have been relatively limited. Near-aphelion observations by Meech (1996) and Licandro et al. (2000) indicated that it has a large (equivalent radius of $3.2 \mathrm{~km}$ ) and elongated (axial ratio $>1.4$ ) nucleus, and they suggested a rotation period of $6.38 \mathrm{hr}$ (see also Lamy et al. 2004). Sekanina (1990) analyzed various publicly available data sets and concluded that it has very low activity for its size, suggesting that this likely hampered an earlier discovery. Most intriguingly, Langland-Shula \& Smith (2007) and Schleicher (2008) found it to be depleted in $\mathrm{C}_{2}$ and $\mathrm{C}_{3}$ and highly depleted in CN, with Schleicher (2008) suggesting this combination is primordial and possibly due to an interstellar origin.

The current work is motivated by our interest in the properties of objects with low perihelion distances. Despite the aforementioned challenging viewing geometry, $96 \mathrm{P}$ is the most accessible comet on a low- $q$ orbit, potentially allowing evolutionary effects to be detected on short timescales. Its 
Table 1

Summary of Comet 96P/Machholz 1 Observations and Geometric Parameters during Our 2017/18 Observations ${ }^{\mathrm{a}}$

\begin{tabular}{|c|c|c|c|c|c|c|c|c|c|c|c|}
\hline UT Date $^{\mathrm{b}}$ & UT Range & $\Delta \mathrm{T}$ (days) $^{\mathrm{c}}$ & Tel. $^{\mathrm{d}}$ & $r_{\mathrm{H}}(\mathrm{au})$ & $\Delta(\mathrm{au})$ & $\mathrm{PA}\left({ }^{\circ}\right)^{\mathrm{e}}$ & $\alpha\left({ }^{\circ}\right)^{\mathrm{f}}$ & $\sigma_{\mathrm{m}}^{\mathrm{g}}(\mathrm{mag})$ & $\sigma_{\text {cal }}^{\mathrm{h}}(\mathrm{mag})$ & Filters & $\overline{\text { Conditions }}$ \\
\hline 2017 Apr 7 & $08: 22-09: 38$ & -203.6 & SOAR & 3.179 & 3.393 & 240.7 & 17.1 & 0.021 & 0.07 & $g^{\prime}, r^{\prime}, i^{\prime}, z^{\prime}$ & cirrus \\
\hline 2017 Jul 2 & $02: 43-10: 45$ & -117.7 & SOAR & 2.283 & 1.537 & 289.0 & 21.3 & 0.004 & 0.02 & $g^{\prime}, r^{\prime}, i^{\prime}, z^{\prime}$ & photometric \\
\hline 2017 Jul 3 & 08:38-09:11 & -116.6 & SOAR & 2.270 & 1.520 & 290.7 & 21.3 & 0.003 & 0.03 & $r^{\prime}$ & photometric \\
\hline 2018 Jun 25 & 00:02-09:19 & +240.2 & SOAR & 3.493 & 2.477 & 294.0 & 0.6 & 0.027 & 0.08 & $g^{\prime}, r^{\prime}, i^{\prime}, z^{\prime}$ & cirrus \\
\hline 2018 Jul 10/11 & $23: 21-04: 32$ & +256.1 & SOAR & 3.619 & 2.642 & 85.4 & 5.2 & 0.028 & 0.03 & $r^{\prime}, i^{\prime}, z^{\prime}$ & cirrus \\
\hline 2018 Jul 12 & $02: 41-07: 17$ & +257.2 & NTT & 3.628 & 2.657 & 85.8 & 5.5 & 0.080 & 0.11 & $r^{\prime}$ & clear \\
\hline 2018 Jul 31/Aug 1 & $22: 03-01: 35$ & +277.0 & WHT & 3.778 & 2.980 & 90.0 & 10.7 & 0.041 & 0.03 & $r^{\prime}$ & clear \\
\hline 2018 Aug $1 / 2$ & $21: 49-01: 29$ & +278.0 & WHT & 3.784 & 2.998 & 90.1 & 10.9 & 0.029 & 0.02 & $r^{\prime}$ & photometric \\
\hline 2018 Aug 2 & $21: 47-22-51$ & +279.0 & WHT & 3.792 & 3.017 & 90.3 & 11.1 & 0.073 & 0.09 & $r^{\prime}$ & photometric \\
\hline
\end{tabular}

Notes.

a All parameters were taken at the midpoint of each night's observations.

${ }^{\mathrm{b}}$ UT date of the observations (note that some nights span multiple UT dates).

${ }^{\mathrm{c}}$ Time since perihelion (2017 October 27.96).

$\mathrm{d}$ Telescope: SOAR = Southern Astrophysical Research $(4.2 \mathrm{~m})$, NTT = New Technology Telescope $(3.54 \mathrm{~m})$, WHT = William Herschel Telescope $(4.2 \mathrm{~m})$.

e Position angle (PA) of the Sun.

${ }^{\mathrm{f}}$ Phase angle.

g Average photometric (statistical) uncertainty in the $r^{\prime}$-band magnitudes calculated for the night.

${ }^{\mathrm{h}}$ Uncertainty in the absolute calibration of the $r^{\prime}$-band magnitudes for the night.

perihelion distance is less than half that of $2 \mathrm{P} /$ Encke's $(q=0.336 \mathrm{au})$, the comet typically most associated with evolutionary effects (e.g., Sekanina 1988, 1991). The only short-period comet with a perihelion distance smaller than $96 \mathrm{P}$ 's to be observed from the ground is $322 \mathrm{P} / \mathrm{SOHO} 1$ $(q=0.054 \mathrm{au})$, but $322 \mathrm{P}$ is much smaller and intrinsically fainter. Knight et al. (2016) found that 322P has unusual properties: it was inactive near 1 au despite behavior consistent with having a coma in the $\mathrm{SOHO}$ fields of view (Lamy et al. 2013), it has a fast rotation period and high albedo suggestive of an asteroidal rather than cometary origin, and it has nucleus colors atypical for comet nuclei. Since 96P is unquestionably cometary, a comparison of it with $322 \mathrm{P}$ may yield insight into whether 322P's properties are evolutionary or primordial. This will aid our understanding of the low- $q$ asteroid population. Granvik et al. (2016) found a significant deficit of asteroids on small perihelion distance orbits, which they suggested was due to the preferential loss of low-albedo objects via disruption on timescales of less than $250 \mathrm{yr}$.

Although the cause is in doubt, 96P already demonstrates behavior of disruption. Two groups of short-period "sunskirting" comets, the Marsden and Kracht groups, were discovered in SOHO LASCO images (see Marsden 2005; Battams \& Knight 2017; Jones et al. 2018 and references therein). Their orbits were recognized to be similar to future orbits of 96P, and they have been dynamically linked to 96P, along with several meteor streams, as part of the "Machholz Complex" (Ohtsuka et al. 2003; Sekanina \& Chodas 2005). The Machholz Complex appears to be continuing to evolve, with some Kracht and Marsden objects failing to be reobserved and new fragments apparently being produced (e.g., Knight 2008).

During its last two perihelion passages, 96P itself has been accompanied by two (in 2012) and three (in 2017) faint fragments when seen in SOHO LASCO images (Battams \& Lui 2013, Battams $\left.^{8,9}\right)$. Due to the short orbital arcs and resulting large ephemeris uncertainties, it is not certain when these fragments

\footnotetext{
8 https://sungrazer.nrl.navy.mil/index.php? $\mathrm{p}=$ news/machholz_babies

9 https://twitter.com/sungrazercomets/status/925042636460785665
}

were produced (Sekanina 2013) or even if the same objects were reobserved in 2017 , but it is clear that $96 \mathrm{P}$ is a prime target for continued monitoring. Despite the small perihelion distance, 96P is still well beyond the Roche limit for a typical cometary density (Knight \& Walsh 2013), and tidal forces are not likely to be the primary cause of its ongoing fragmentation (Sekanina \& Chodas 2005). Mueller \& Samarasinha (2018) argued that it is among the comets most likely to exhibit a large change in rotation period due to torques caused by outgassing. As we will show later, 96P's rotation period is close to the rotational spin limit for a strengthless body, so disruption due to rotational spin-up is viable and potentially detectable on short timescales.

We took advantage of 96P's large and relatively inactive nucleus to study it before and after the most recent perihelion passage (2017 October 27.96), allowing us to obtain a highprecision nucleus lightcurve with modest (4 m class) telescope apertures. We obtained observations on three nights in 2017 and six nights in 2018 (Section 2). We did not detect a coma or tail, and we measured nucleus properties such as size and color (Section 3) and searched for nearby fragments (Section 4). In Section 5 we present detailed lightcurve analyses, including highly constrained rotation periods pre- and post-perihelion that allow us to look for a change in rotation period. Finally, we discuss and summarize our results in Sections 6 and 7, respectively.

\section{Observations and Reductions}

\subsection{Observing Overview and Reduction}

Images were obtained on a total of nine nights during the $2017 / 2018$ apparition, primarily using the broadband $r^{\prime}$ filter. The observations were always tracked at the ephemeris rate of the comet, with the exception of the images obtained on 2018 July $11 / 12$ with the New Technology Telescope, which were taken with sidereal tracking and short-enough exposures to prevent the comet from moving more than the seeing disk. A summary of the observations, filters used, and weather conditions is given in Table 1. Due to the wide range of telescopes, instruments, and reduction techniques that were 

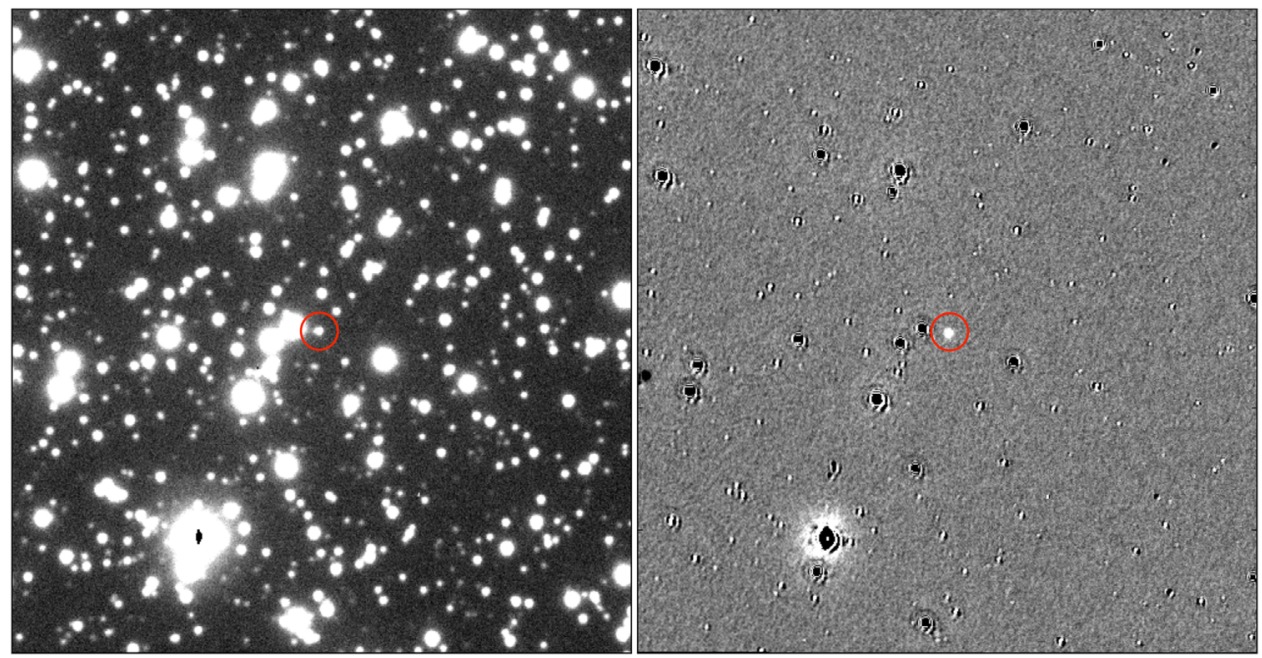

Figure 1. DIA image processing of the same initial image (left), taken on the night of 2018 July 10/11. The images are approximately $300,000 \mathrm{~km}$ across at the scale of the comet. The position of the comet is shown by the red circle. The star in the lower left-hand corner in each image could not be removed properly because it was saturated.

implemented, the individual observation runs are described individually in the subsections to follow. Prior to any calibrations, all data were reduced using standard bias and flat-field techniques. Frames that were significantly contaminated by field stars were discarded. Coherent lightcurve displaying at least one peak and trough were obtained on a total of four nights on 2017 July 2, 2018 July 10/11, 2018 July 31 /August 1, and 2018 August 2. On the remaining five nights of observations, we acquired snapshots of comet $96 \mathrm{P}$ at one or more epochs during a night.

\subsubsection{April 7}

Images taken on 2017 April 7 were obtained using the $4.2 \mathrm{~m}$ Southern Astrophysical Research (SOAR) telescope on Cerro Pachon in Chile using the Goodman Spectrograph Red Camera, which utilizes an e2v 231-84 CCD (Clemens et al. 2004). On-chip $2 \times 2$ binning produced images with a pixel scale of $0.30 \operatorname{arcsec}_{\text {pixel }}^{-1}$. We obtained snapshot observations using Sloan Digital Sky Survey (SDSS) $g^{\prime}, r^{\prime}, i^{\prime}$, and $z^{\prime}$ (e.g., Fukugita et al. 1996). The conditions were not photometric, and the field was too far south for the PanSTARRS catalog, so absolute calibrations were performed by reimaging the field on 2017 July 2 (when it was photometric) in the same four broadband filters. We corrected for the varying extinction using on-chip comparison stars, finding typical corrections of $\sim 0.5 \mathrm{mag}$, which is near the limit of what we have previously found to yield reliable lightcurve (Knight et al. 2012; Eisner et al. 2017).

\subsubsection{July 2-3}

Images taken on 2017 July 2 and 3 were obtained at SOAR with the SOAR Optical Imager (SOI), ${ }^{10}$ which uses a mosaic of two e2v $2048 \times 4096$ pixel CCDs (Walker et al. 2003). On-chip $2 \times 2$ binning produced images with a pixel scale of 0.154 arcsec pixel $^{-1}$. On July 2 we monitored the comet all night in $r^{\prime}$ and occasionally took snapshots using the $g^{\prime}, i^{\prime}$, and $z^{\prime}$ filters. A short sequence of $r^{\prime}$ images was obtained on July 3. The conditions throughout both of these nights were

\footnotetext{
${ }_{10}$ SOI was used during this run to support the blue wavelength sensitivity needed by another program on these nights.
}

photometric, allowing us to use SDSS standard stars to perform absolute calibrations (Smith et al. 2002).

\subsubsection{June 25 and 2018 July 10/11}

The observations on 2018 June 25 and July 10/11 made use of the Goodman Spectrograph Red camera at the SOAR telescope. On both nights, images were obtained using broadband $g^{\prime}, r^{\prime}, i^{\prime}$, and $z^{\prime}$ filters, but, due to technical problems, the $g^{\prime}$ filter images obtained on 2018 July 10/11 could not be used for further analysis.

Throughout our 2018 observations, the comet was located in the midst of the Milky Way and, therefore, suffered from significant contamination from crowded star fields. Difference image analysis (DIA; Bramich 2008; Bramich et al. 2013) was implemented to recover useful data by removing background stars, as we previously used to extract useful data on $67 \mathrm{P} /$ Churyumov-Gerasimenko when it was located in crowded fields in 2014 (Snodgrass et al. 2016). The technique uses background templates (or reference images) of the fields, which were taken on 2018 July 11, and works by blurring them to match the seeing of each comet frame. The blurred reference image is then subtracted from the comet frames, removing the majority of the background stars, as shown in Figure 1. The figure also demonstrates that saturated stars cannot be removed. Absolute calibration was carried out by measuring field stars in the reference image and comparing the observed magnitudes to those from the Pan-STARRS catalog PS1 Data Release 1 (Chambers 2017). The PS1 filter system differs from SDSS by small amounts, so we corrected for this using the equations presented by Tonry et al. (2012).

We monitored the comet in $r^{\prime}$ on both nights and took three sequences of $g^{\prime}, r^{\prime}, i^{\prime}$, and $z^{\prime}$ filter images on 2018 July 10/11 at times when the comet was located between field stars. Because reference images were only taken in $r^{\prime}$, the DIA method could not be applied to these data. We determined absolute calibrations on each image using the Pan-STARRS catalog.

\subsubsection{July 12}

Snapshot $r^{\prime}$-band images were obtained on 2018 July 12 using the $3.54 \mathrm{~m}$ New Technology Telescope (NTT) at La Silla, Chile, with the ESO Faint Object Spectrograph and Camera 
Table 2

Example Observed $r^{\prime}$-band Magnitudes and Their Statistical Uncertainties

\begin{tabular}{|c|c|c|c|c|c|c|c|c|c|c|c|}
\hline Date $^{a}$ & $\overline{\mathrm{UT}^{\mathrm{b}}}$ & $\overline{m_{r^{\prime}}{ }^{\mathrm{c}}}$ & $\overline{\sigma_{r^{\prime}}{ }^{\mathrm{d}}}$ & Date $^{a}$ & $\overline{\mathrm{UT}^{\mathrm{b}}}$ & $\overline{m_{r^{\prime}}{ }^{\mathrm{c}}}$ & $\overline{\sigma_{r^{\prime}}{ }^{\mathrm{d}}}$ & Date $^{a}$ & $\overline{\mathrm{UT}^{\mathrm{b}}}$ & $\overline{m_{r^{\prime}}{ }^{\mathrm{c}}}$ & $\sigma_{r^{\prime}}^{\mathrm{d}}$ \\
\hline 2017 Apr 7 & $08: 22: 04$ & 20.458 & 0.053 & 2017 Jul 2 & 04:05:57 & 18.498 & 0.005 & 2017 Jul 2 & $05: 35: 27$ & 18.602 & 0.004 \\
\hline 2017 Apr 7 & 08:23:09 & 20.489 & 0.022 & 2017 Jul 2 & 04:08:06 & 18.450 & 0.004 & 2017 Jul 2 & $05: 37: 36$ & 18.584 & 0.004 \\
\hline 2017 Apr 7 & $08: 27: 05$ & 20.474 & 0.022 & 2017 Jul 2 & $04: 10: 15$ & 18.423 & 0.004 & 2017 Jul 2 & $05: 39: 45$ & 18.526 & 0.004 \\
\hline 2017 Apr 7 & $08: 34: 17$ & 20.467 & 0.021 & 2017 Jul 2 & $04: 12: 23$ & 18.382 & 0.004 & 2017 Jul 2 & $05: 41: 53$ & 18.536 & 0.004 \\
\hline 2017 Apr 7 & 09:00:45 & 20.465 & 0.021 & 2017 Jul 2 & $04: 14: 32$ & 18.346 & 0.004 & 2017 Jul 2 & $05: 44: 02$ & 18.524 & 0.004 \\
\hline
\end{tabular}

Notes.

${ }^{\text {a }}$ UT date of observation.

${ }^{\mathrm{b}} \mathrm{UT}$ at midpoint of the exposure.

c Observed $r^{\prime}$-band magnitude.

${ }^{\mathrm{d}}$ Statistical uncertainty in $r^{\prime}$-band magnitude.

(This table is available in its entirety in machine-readable form.)

(EFOSC2), which employs a $2048 \times 2048$ pixel Loral/Lesser CCD (Buzzoni et al. 1984). On-chip $2 \times 2$ binning produced images with a pixel scale of $0.24 \operatorname{arcsec}_{\text {pixel }}{ }^{-1}$. The PanSTARRS catalog was used to perform absolute calibrations. Because conditions were not photometric, we monitored the brightness of seven field stars in order to correct for variations in transparency throughout the night (see methodology by Eisner et al. 2017 for details), yielding image-to-image corrections between -0.01 and 0.13 mag.

\subsubsection{July 31-August 2}

Our final images were acquired using the $4.2 \mathrm{~m}$ William Herschel Telescope (WHT), in La Palma, Spain, on 2018 July 31 through August 2. These observations made use of the auxiliary port camera (ACAM; Benn et al. 2008), which employs an e2v $2 \mathrm{k} \times 4 \mathrm{k}$ CCD. On-chip binning of $1 \times 1$ yielded a pixel scale of 0.253 arcsec pixel ${ }^{-1}$.

On the first two of these nights, the comet observations suffered from significant contamination from background stars, so DIA methods were applied in order to obtain coherent lightcurves. This was not necessary on 2018 August 2 because the comet was fortuitously located in front of a Milky Way dust lane, producing a field of view relatively free of stars. Field stars in the Pan-STARRS catalog were used to perform absolute calibrations on all three nights.

\subsection{Comet Measurements}

Following the methodology of earlier papers (e.g., Knight et al. 2011; Eisner et al. 2017), we extracted the flux by centroiding on the comet nucleus and integrating inside a circular aperture. The aperture radii were independently chosen for each night to maximize the signal-to-noise ratio $(\mathrm{S} / \mathrm{N})$ while minimizing the contamination from field stars, but were typically around 1.5 times the typical FWHM. On nights where the DIA methods were applied to extract data (2018 June 24, July 10/11, July 31/August 1, August 1/2), the aperture sizes were automatically adjusted throughout the night in order to match the seeing, with an aperture radius set equal to the pointspread function FWHM to maximize the $\mathrm{S} / \mathrm{N}$, and an aperture correction was applied (following the procedure applied by, e.g., Snodgrass et al. 2005, 2013). The median sky flux, calculated in an annulus with a radius significantly larger than the circular aperture, was subsequently subtracted from the measured flux of the comet. All observed $r^{\prime}$-band magnitudes and their statistical uncertainties are tabulated and available as an online supplement, and an example observations are shown in Table 2. As will be discussed in the following section, we did not detect any coma, so the absolute magnitude (apparent magnitude reduced to unit heliocentric and geocentric distances at zero solar phase angle), $H$, was calculated using standard asteroidal normalization (e.g., Jewitt 1991), assuming a linear phase correction of 0.04 mag per degree.

\section{Nucleus Properties}

\subsection{Assessment of Coma}

In order to study properties of the nucleus, the extent of the contribution from the coma needed to be assessed. Individual images on all nights showed no evidence of a prominent coma or tail, so we created stacked images using a median of all the frames obtained on a single night. An example of this is shown in the left-hand panel of Figure 2 for the night of 2017 July 1 with a total integration time of 20,280 s (5.6 hr). These stacked images also showed no signs of tail or coma.

Additionally, we compared the radial profiles of stacked images and single-frame images of the comet to radial profiles of nearly untrailed field stars. All three were found to be consistent with one another in both our pre- and post-perihelion observations, further confirming that we were observing a bare nucleus. A representative example is shown in the right-hand panel of Figure 2 for the night of 2017 July 1.

\subsection{Nucleus Size}

Based on the assumption that we observed a bare nucleus, we estimated its effective radius $\left(R_{n}\right)$ from the absolute magnitude using the standard methodology devised by Russell (1916) and reformulated by Lamy et al. (2004):

$$
R_{n}=\frac{1.496 \times 10^{11}}{\sqrt{p}} 10^{0.2\left(m_{\odot}-H\right)}
$$

where $p, H$, and $m_{\odot}$ are the geometric albedo, absolute magnitude, and the apparent magnitude of the Sun, respectively, and $R_{n}$ has units of meters. A value of -26.93 was used for the $r^{\prime}$-band magnitude of the Sun, based on the color of the Sun $(V-R=0.370$; Colina et al. 1996) and the Smith et al. (2002) $V$ to $r^{\prime}$ transformation. Typical values for albedo (0.04) and nucleus phase angle correction (0.04 mag deg ${ }^{-1}$; see Section 3.4) were assumed (e.g., Lamy et al. 2004). The 

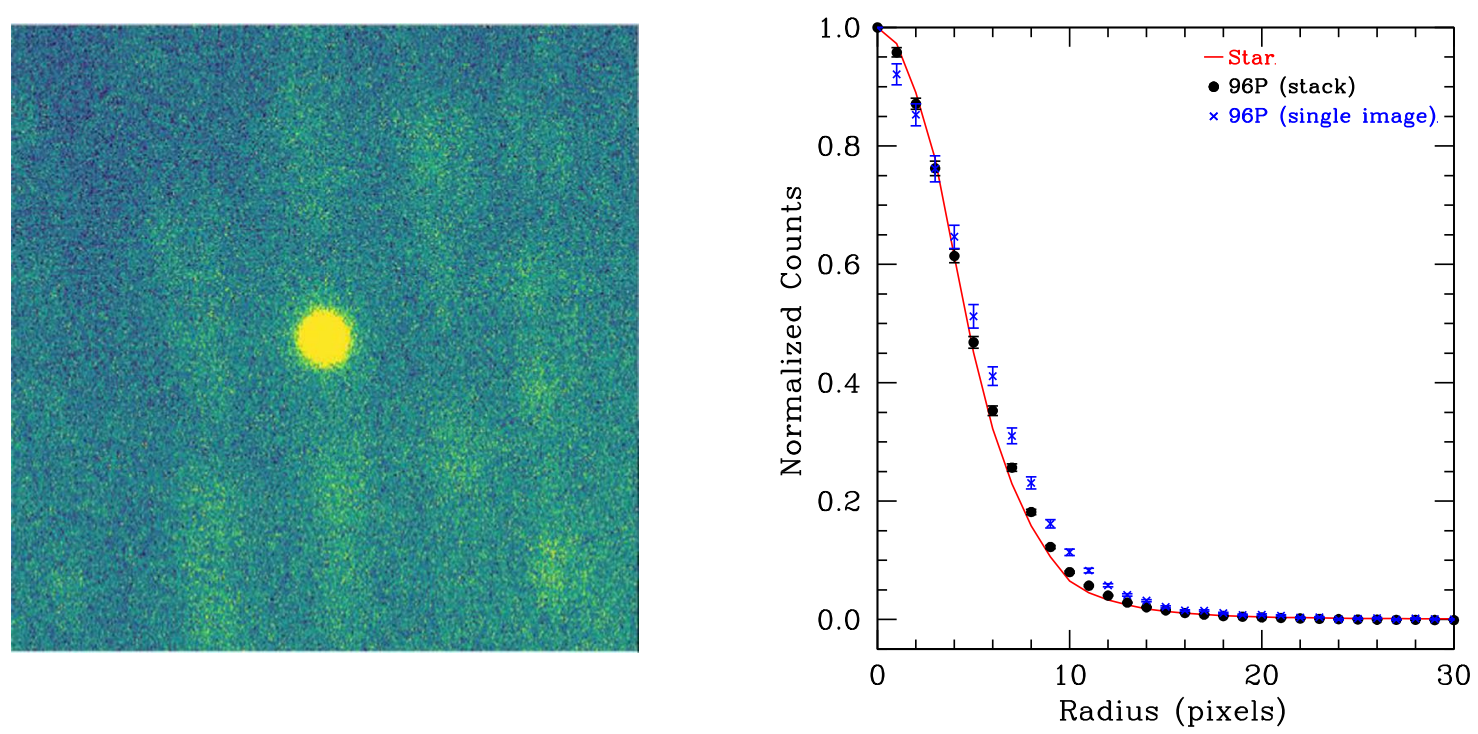

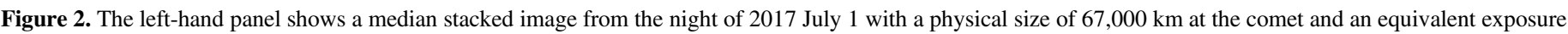

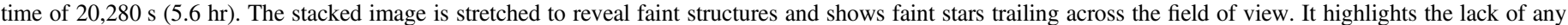

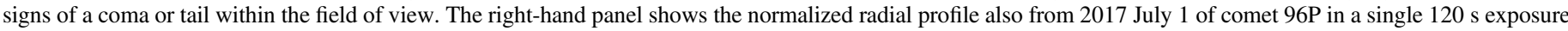

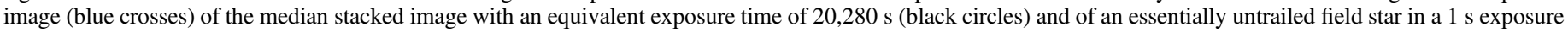
(red line). The profile of the comet on the single image is slightly wider than the nightly stack because of variations in seeing.

Table 3

Summary of Comet 96P/Machholz 1's Average Nucleus Properties

\begin{tabular}{lcccc}
\hline \hline Date $^{\mathrm{a}}$ & $\overline{\boldsymbol{H}}_{\boldsymbol{\prime}^{\prime}} \mathrm{b}^{\mathrm{b}}$ & $\boldsymbol{R}_{\mathrm{n}}(\mathrm{km})^{\mathrm{c}}$ & Amplitude $^{\mathrm{d}}$ & Axis ratio $^{\mathrm{e}}$ \\
\hline 2017 Apr 7 & $14.5 \pm 0.1$ & $3.87 \pm 0.2$ & $\ldots$ & $\ldots$ \\
2017 Jul 2 & $14.85 \pm 0.05$ & $3.30 \pm 0.08$ & $0.50 \pm 0.05$ & $1.58 \pm 0.07$ \\
2017 Jul 3 & $14.85 \pm 0.10$ & $3.30 \pm 0.15$ & $\ldots$ & $\ldots$ \\
2018 Jun 25 & $14.55 \pm 0.1$ & $3.78 \pm 0.2$ & $\ldots$ & $\ldots$ \\
2018 Jul 10 & $14.85 \pm 0.05$ & $3.29 \pm 0.08$ & $0.30 \pm 0.10$ & $1.32 \pm 0.12$ \\
2018 Jul 12 & $15.0 \pm 0.1$ & $3.1 \pm 0.1$ & $\ldots$ & $\ldots$ \\
2018 Jul 31 & $14.80 \pm 0.05$ & $3.37 \pm 0.08$ & $0.35 \pm 0.10$ & $1.38 \pm 0.13$ \\
2018 Aug 1 & $14.80 \pm 0.05$ & $3.37 \pm 0.08$ & $0.35 \pm 0.10$ & $1.38 \pm 0.13$ \\
2018 Aug 2 & $14.80 \pm 0.10$ & $3.37 \pm 0.15$ & $\cdots$ & $\cdots$ \\
\hline
\end{tabular}

Notes.

${ }^{\mathrm{a}}$ UT date at the start of the observations.

$\mathrm{b}$ Estimated absolute magnitude at midpoint of the magnitude variation.

${ }^{\mathrm{c}}$ Estimated nucleus radius.

d The maximum peak-to-trough amplitude for nights that yielded a clear peak and trough.

e Minimum axis ratio.

average absolute magnitudes $\left(\bar{H}_{r^{\prime}}\right)$ and corresponding uncertainties are given in Table 3. For nights with both a peak and a trough, they were determined at the midpoint of the magnitude variation. On nights with incomplete or ambiguous lightcurves the average absolute magnitude was estimated based on the rotational phasing (see Section 5). The average nucleus radius across all nights was found to be $3.41 \pm 0.24 \mathrm{~km}$, where the uncertainty is the standard deviation of the nightly results.

On nights where the broadband $r^{\prime}$ data showed a clear peak and trough, the minimum axial ratio of the nucleus was determined based on the peak-to-trough maximum amplitude of the lightcurve. The peak-to-trough variation of the lightcurves and the corresponding axial ratios are tabulated in Table 3 .

Both the average radius and axial ratio were compared to previously determined values. Licandro et al. (2000) observed a "star-like" nucleus at $r_{H}=4.89$ au in 1995, finding an equivalent radius of $3.6 \mathrm{~km}$ and axial ratio of 1.4 . Meech (1996) reports an equivalent radius of $2.8 \mathrm{~km}$ and an axial ratio of 1.4 but provides no further information. Using these two reports, Lamy et al. (2004) note that an axial ratio of $a / b \sim 1.5$ could yield a spheroid with $a=4.3 \mathrm{~km}$ and $b=2.8 \mathrm{~km}$, making both observations consistent with our $R_{n}$.

\subsection{Nucleus Color}

We obtained color measurements pre- and post-perihelion on the nights of 2017 April 7, 2017 July 2, and 2018 July 10/11. On 2017 April 7, color observations, following the sequence $g^{\prime}$, $r^{\prime}, i^{\prime}, z^{\prime}, z^{\prime}, i^{\prime}, r^{\prime}, g^{\prime}$, were taken twice throughout the night. For each sequence, the mean magnitude per filter was used to calculate quasi-simultaneous colors $g^{\prime}-r^{\prime}, r^{\prime}-i^{\prime}$, and $i^{\prime}-z^{\prime}$. On 2017 July $1, r^{\prime}$-band observations were interleaved with observations in the $g^{\prime}, i^{\prime}$, and $z^{\prime}$ filters five times throughout the night. We interpolated the $r^{\prime}$-band observations to the time of the respective $g^{\prime}, i^{\prime}$, and $z^{\prime}$ observations to calculate colors, but the resulting $g^{\prime}-r^{\prime}, r^{\prime}-i^{\prime}$, and $i^{\prime}-z^{\prime}$ are not quasisimultaneous. As previously mentioned, we were unable to obtain usable images in the $g^{\prime}$ filter on the night of 2018 July $10 / 11$, so we were only able to calculate $r^{\prime}-i^{\prime}$ and $i^{\prime}-z^{\prime}$ colors post-perihelion.

These colors were converted to $B-V, V-R$, and $R-I$ colors using translations from Lupton (2005) to allow for direct comparisons with existing data sets. Photometric uncertainties were calculated from adding in quadrature the photon statistics (see column 9 of Table 1), uncertainties in the absolute calibration (see column 10 of Table 1), and uncertainties in the color translations (typically less than 0.01 ). All color measurements and their uncertainties are tabulated in Table 4.

Average colors were determined for each night in order to compare the color of 96P's nucleus to that of other known comet nuclei colors. The average colors are tabulated in Table 4, where the uncertainties are the standard deviations of all the individual color measurements on a given night. It should be noted that the uncertainties in the individual 
Table 4

Summary of Comet 96P/Machholz 1 Color Measurements

\begin{tabular}{|c|c|c|c|c|c|c|c|c|c|c|c|}
\hline UT Date & Time & $g^{\prime}$ & $r^{\prime}$ & $i^{\prime}$ & $z^{\prime}$ & $g^{\prime}-r^{\prime}$ & $\overline{r^{\prime}-i^{\prime}}$ & $\overline{i^{\prime}-z^{\prime}}$ & $\overline{B B-V^{a}}$ & $\overline{V-R^{\mathrm{a}}}$ & $\overline{R-I^{\mathrm{a}}}$ \\
\hline \multirow[t]{2}{*}{2017 Apr 7} & $08: 47$ & $20.90 \pm 0.069$ & $20.47 \pm 0.047$ & $20.30 \pm 0.054$ & $20.23 \pm 0.087$ & $0.43 \pm 0.083$ & $0.17 \pm 0.071$ & $0.07 \pm 0.102$ & $0.62 \pm 0.08$ & $0.35 \pm 0.08$ & $0.39 \pm 0.07$ \\
\hline & 09:24 & $20.75 \pm 0.069$ & $20.29 \pm 0.042$ & $20.14 \pm 0.051$ & $20.08 \pm 0.074$ & $0.46 \pm 0.081$ & $0.15 \pm 0.066$ & $0.07 \pm 0.090$ & $0.64 \pm 0.08$ & $0.37 \pm 0.08$ & $0.38 \pm 0.07$ \\
\hline Average $^{b}$ & & & & & & $\mathbf{0 . 4 5} \pm \mathbf{0 . 0 2}$ & $\mathbf{0 . 1 6} \pm \mathbf{0 . 0 1}$ & $\mathbf{0 . 0 7} \pm \mathbf{0 . 0 1}$ & $\mathbf{0 . 6 3} \pm \mathbf{0 . 0 1}$ & $\mathbf{0 . 3 6} \pm \mathbf{0 . 0 1}$ & $\mathbf{0 . 3 9} \pm \mathbf{0 . 0 1}$ \\
\hline \multirow[t]{5}{*}{2017 Jul 1} & $03: 54$ & $19.10 \pm 0.017$ & $18.56 \pm 0.016$ & $18.41 \pm 0.016$ & $18.28 \pm 0.019$ & $0.54 \pm 0.023$ & $0.13 \pm 0.023$ & $0.11 \pm 0.025$ & $0.71 \pm 0.03$ & $0.42 \pm 0.03$ & $0.36 \pm 0.02$ \\
\hline & 05:02 & $18.94 \pm 0.016$ & $18.42 \pm 0.016$ & $18.27 \pm 0.016$ & $18.32 \pm 0.019$ & $0.52 \pm 0.022$ & $0.18 \pm 0.022$ & $-0.02 \pm 0.025$ & $0.70 \pm 0.03$ & $0.41 \pm 0.03$ & $0.41 \pm 0.02$ \\
\hline & $06: 36$ & $18.90 \pm 0.016$ & $18.4 \pm 0.015$ & $18.17 \pm 0.016$ & $18.15 \pm 0.017$ & $0.50 \pm 0.022$ & $0.23 \pm 0.022$ & $0.03 \pm 0.023$ & $0.68 \pm 0.02$ & $0.40 \pm 0.02$ & $0.46 \pm 0.02$ \\
\hline & $07: 44$ & $19.05 \pm 0.016$ & $18.54 \pm 0.015$ & $18.39 \pm 0.016$ & $18.31 \pm 0.018$ & $0.51 \pm 0.022$ & $0.17 \pm 0.022$ & $0.09 \pm 0.024$ & $0.69 \pm 0.02$ & $0.40 \pm 0.02$ & $0.40 \pm 0.02$ \\
\hline & 09:03 & $18.88 \pm 0.016$ & $18.37 \pm 0.015$ & $18.23 \pm 0.016$ & $18.18 \pm 0.017$ & $0.51 \pm 0.022$ & $0.18 \pm 0.022$ & $0.09 \pm 0.023$ & $0.69 \pm 0.02$ & $0.40 \pm 0.02$ & $0.41 \pm 0.02$ \\
\hline Average $^{\mathrm{b}}$ & & & & & & $\mathbf{0 . 5 2} \pm \mathbf{0 . 0 2}$ & $0.18 \pm 0.04$ & $0.06 \pm 0.06$ & $\mathbf{0 . 6 9} \pm \mathbf{0 . 0 1}$ & $\mathbf{0 . 4 1} \pm \mathbf{0 . 0 1}$ & $\mathbf{0 . 4 1} \pm \mathbf{0 . 0 4}$ \\
\hline
\end{tabular}

Notes. The boldface indicates the nightly averages.

${ }^{a} B-V, V-R$, and $R-I$ colors were calculated using translations from Lupton (2005).

$\mathrm{b}$ The mean color on a given night. 

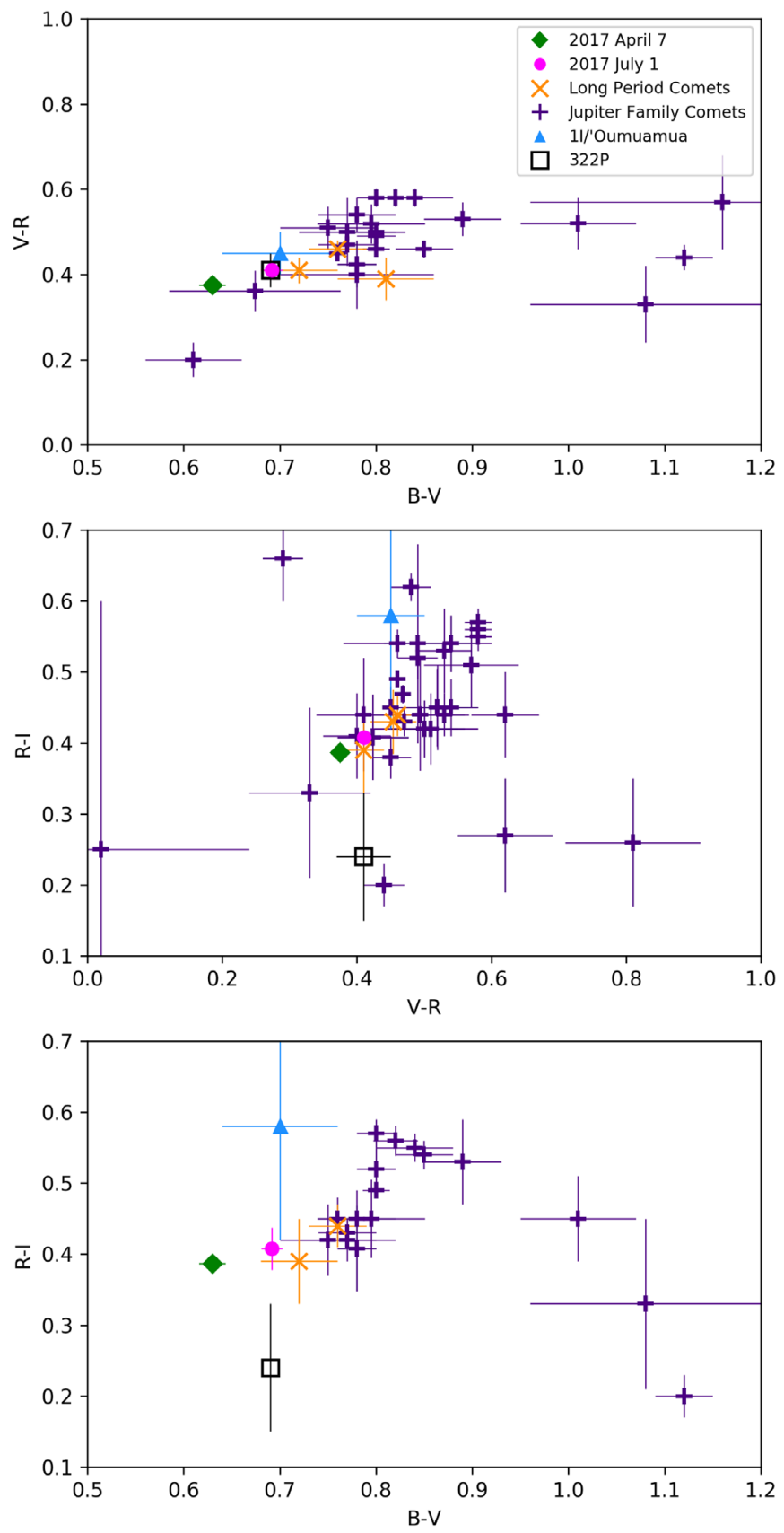

Figure 3. Average colors for $96 \mathrm{P}$ (pink circle and green diamond) compared to colors of long-period comets (yellow crosses), Jupiter-family comets (indigo plus signs), 1I/Oumuamua (light blue triangle), and 322P (black squares). See text for references. Our SDSS colors for 96P were translated to Johnson colors following Lupton (2005) for comparison with the other data sets. Some of the comets do not have all three color measurements and therefore only show up in one out of the three plots.

measurements, which are also given in Table 4, are mostly substantially larger. The $V-R$ color of comet $96 \mathrm{P}$ had previously been measured to be $0.3 \pm 0.1$ during the $1995 /$ 1996 apparition (Licandro et al. 2000) and $0.429 \pm 0.027$ (apparition unspecified; Meech et al. 2004). Both of these values are consistent with our $V-R$ colors determined for the $2017 / 2018$ apparition, within the uncertainties of individual measurements.

Figure 3 shows a comparison of the average colors of $96 \mathrm{P}$ to those of the nuclei of Jupiter-family comets and long-period comets (Lamy et al. 2004, 2009; Lamy \& Toth 2009; Hainaut et al. 2012 and references therein), as well as to the interstellar object 1I/'Oumuamua (Bannister et al. 2017; Jewitt et al. 2017) and comet 322P (Knight et al. 2016). The error bars represent the uncertainties calculated from the standard deviation of the measurements, which, as previously mentioned, are significantly smaller than the uncertainties in the individual color measurements. The colors of 96P for the two nights are consistent within the uncertainties of individual images. The $B-V$ and $V-R$ colors presented for 'Oumuamua were determined by Jewitt et al. (2017). Because they did not report on an $R-I$ color, we calculated this value based on data of Bannister et al. (2017), which resulted in significantly larger error bars, as can be seen in Figure 3. Bolin et al. (2018) also reported colors of 'Oumuamua, but, because their uncertainties span the plotted region, we have elected not to show these. The plot shows a notable difference between the color of comet $96 \mathrm{P}$ and the average color of the nuclei of Jupiterfamily comets, exhibiting a generally bluer color. Intriguingly, the plot of $B-V$ versus $V-R$ (top panel of Figure 3) suggests some similarities between the colors of comet $96 \mathrm{P}, 1 \mathrm{I} /$ 'Oumuamua, and $^{\circ}$ $322 \mathrm{P}$, which we will return to in Section 6. The color of 96P is also similar to that of long-period comets (Halley-type comets) in all three color metrics, which will also be discussed in Section 6. The results show no evidence for rotational variation of the colors or for pre-/post-perihelion changes.

\subsection{Nucleus Phase Function}

As our observations spanned a diagnostic range of phase angles from 0.6 to $21^{\circ}$, we attempted to determine the nucleus phase function. We applied two techniques: a simple approach using the estimated midpoint of the magnitude variation each night (as discussed in Section 3.2), and a more sophisticated, stochastic approach that uses all data points and their associated errors (see Kokotanekova et al. 2017, 2018). While the methods yielded results that were generally consistent with each other, the derived phase function slope was highly sensitive to precise absolute calibrations. Due to the overall challenging observations (weather, dense background fields, moon) that necessitated a variety of reduction techniques to process the different nights, we concluded that the absolute calibrations are not known well enough to unambiguously determine the phase function and have elected to use $0.04 \mathrm{mag} \mathrm{deg}^{-1}$ throughout this paper. However, there is some evidence that a flatter phase function $\left(0.02-0.03 \mathrm{mag} \mathrm{deg}^{-1}\right)$ might be valid, which would be consistent with the finding of Kokotanekova et al. (2018) of shallow phase dependences for larger and less active nuclei. There is also some indication of an opposition surge based on the 2018 June 25 data when 96P was close to minimum phase angle $\left(0^{\circ} .6\right)$. While this night was chosen specifically to allow this test, the very dense stellar background in the Milky Way and the strong scattered light from the proximity to a nearly full moon resulted in the largest uncertainties of our data set, and we are thus reluctant to draw a firm conclusion from this night.

\section{Search for Faint Companions}

As discussed in the introduction, 96P has been linked to two groups of near-Sun comets and is believed to have had frequent fragmentation, with $\mathrm{SOHO}$ images of $96 \mathrm{P}$ at perihelion revealing faint accompanying fragments in 2012 (two fragments) and 2017 (three fragments). The fragments seen in 2017 

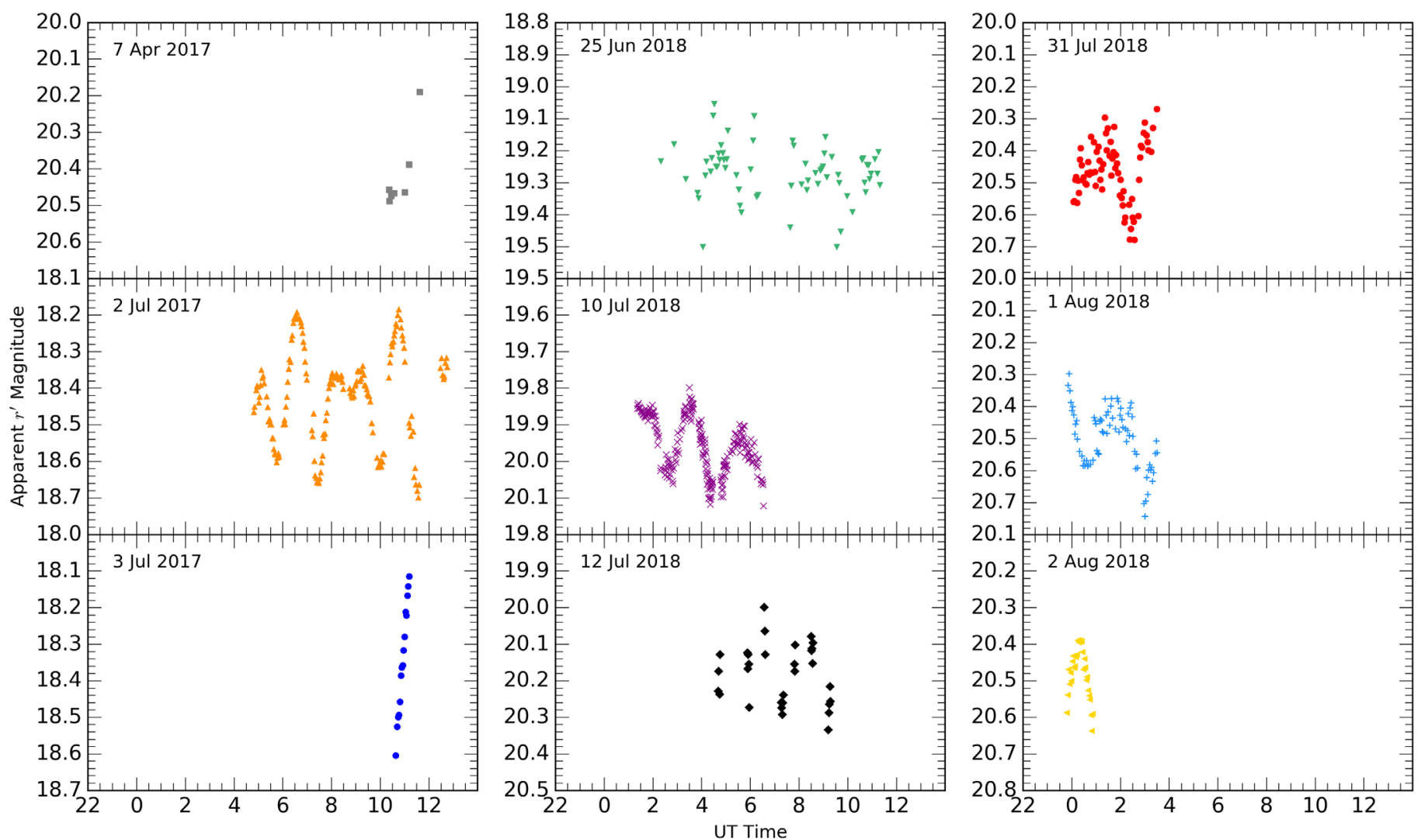

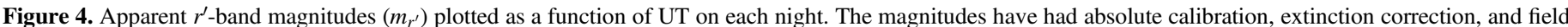

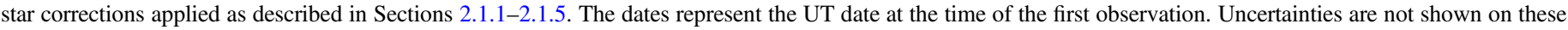

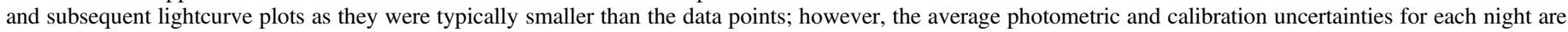
given in Table 1, and the uncertainties of individual measurements are given in the Table 2 .

were on orbits several hours ahead of comet 96P and, therefore, would be expected to be well outside of our fields of view by the time of our ground-based observations in 2018. Nonetheless, if the fragmentation seen by $\mathrm{SOHO}$ in 2017 occurred relatively recently, the fragments could have still been within our field of view during our 2017 ground-based observations. It is also possible that there were fainter or other recently produced fragments in our fields of view.

To search for possible companions, we created deep images by median-combining all images on a given night, effectively removing all stars. The best nights were 2017 July 1 (pre-perihelion) and 2018 July 10/11 (post-perihelion) because they combined a long time on the comet with good seeing and minimal scattered light from the moon. Stacked images on 2017 July 1 totaled $20,280 \mathrm{~s}(5.6 \mathrm{hr})$ over a $10 \times 10 \operatorname{arcsec}$ region centered on the comet (left-hand panel of Figure 2) and $15,360 \mathrm{~s}$ (4.3 hr) over a $30 \times 30 \mathrm{arcsec}$ region, while our 2018 July $10 / 11$ stacked image totaled $15,540 \mathrm{~s}$ (4.3 hr) over a $300 \times 300$ arcsec region centered on the comet. There is a steep decrease in effective exposure time as the comet-centric distance increases for our 2017 data because we conducted large dithers and have restricted the stacked images to only the CCD on which $96 \mathrm{P}$ was observed in case there are small differences in detector sensitivity or calibrations. The 2018 data did not have such problems and consequently go equally deep at all distances.

No faint companions were seen in any of the deep images. We estimated limiting magnitudes in these deep images by scaling the exposure time and $\mathrm{S} / \mathrm{N}$ relative to our individual
96P exposures on each night and setting the detection threshold at an $\mathrm{S} / \mathrm{N}$ of 10 . The 2017 July 1 stacked images rule out any accompanying fragments down to a magnitude of $m_{r^{\prime}} \sim 26$, equivalent to a radius of $\sim 100 \mathrm{~m}$ for the same assumptions as the nucleus within the $30 \times 30$ arcsec region. The 2018 data are severely limited by sky uncertainty, and the 2018 July $10 /$ 11 stacked image only rules out accompanying fragments down to a magnitude of $m_{r^{\prime}} \sim 21$, corresponding to objects with roughly $2 \mathrm{~km}$ radius. As this is nearly as large as 96P itself, searches for newly created accompanying fragments in 2018 are very unconstraining given that estimates of the fragments seen by $\mathrm{SOHO}$ are of the order of a few tens of meters in diameter (Battams \& Knight 2017).

\section{Lightcurve Analysis}

\subsection{Lightcurve Shape}

As shown in Figure 4, the lightcurve is double-peaked with one peak pronounced and the other broad and flat, and one trough significantly shallower and "rounder" than the other. This asymmetry is likely due to the nucleus shape deviating from that of a simple triaxial ellipsoid; for example, it may have sharp edges, large boulders, or flat planes (e.g., Lowry et al. 2012). The asymmetric shape of the lightcurves improved our ability to phase the data as it allowed us to eliminate half-cycle aliases (see Section 5.3).

The pre-perihelion lightcurve is much better defined than the post-perihelion lightcurve because of the significantly higher $\mathrm{S} / \mathrm{N}$ and less-crowded fields. While the general pre- and 


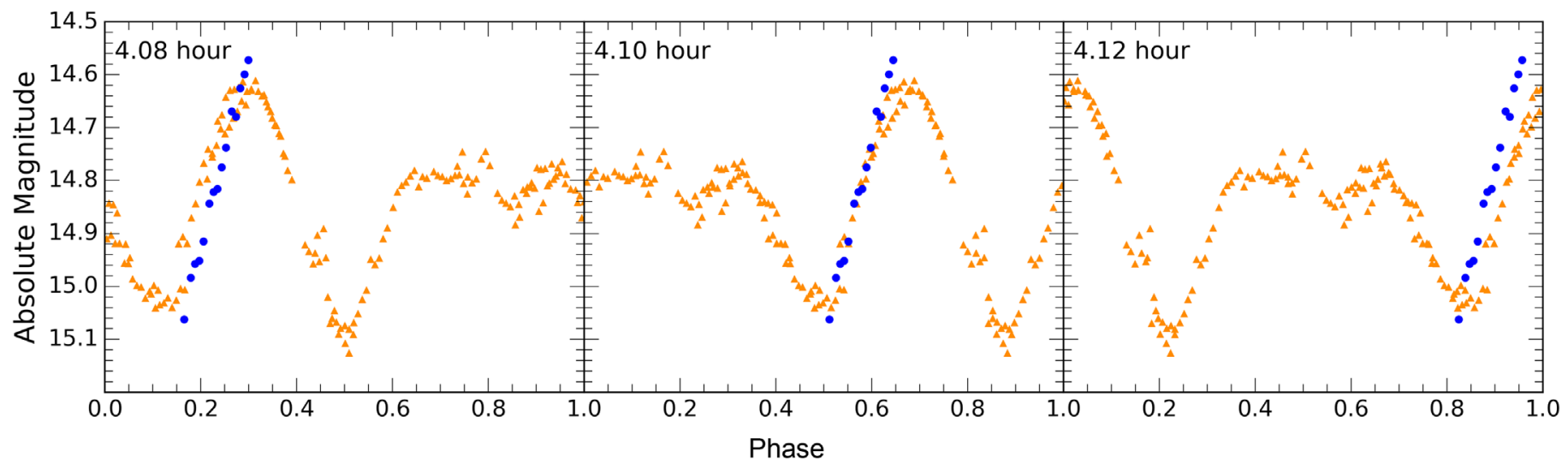

Figure 5. Our reduced pre-perihelion data $\left(H_{r^{\prime}}\right)$ for 2017 July 2 (orange circles) and July 3 (navy blue triangles) phased to $4.08 \mathrm{hr}$ (left), $4.10 \mathrm{hr}$ (middle), and $4.12 \mathrm{hr}$ (right). By iterating though the different periods, we found a best period of $4.10 \pm 0.03 \mathrm{hr}$, where the uncertainty is dominated by the uncertainties in the absolute calibration ( $\pm 0.02 \mathrm{mag})$. Zero phase was set at the time of perihelion (2017 October 27.96).

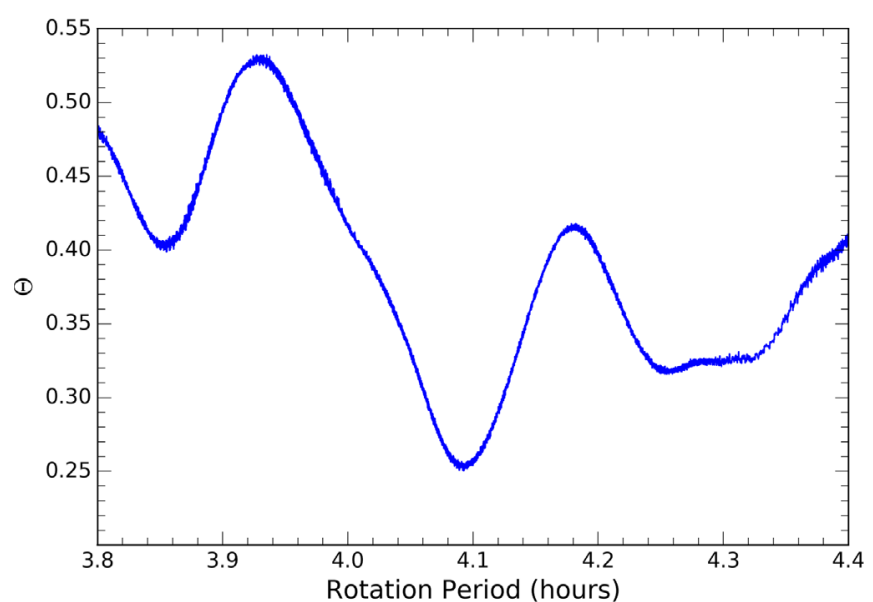

Figure 6. Phase dispersion minimization diagram for the pre-perihelion 2017 July 2 and 3 data. The data curve yields a clear minimum at a period of $4.097 \pm 0.015 \mathrm{hr}$

post-perihelion shape is similar, there are several distinct differences, such as that the flat region is shorter in duration and the amplitude difference between the two peaks is smaller in the post-perihelion data. A likely explanation for these preand post-perihelion differences is that we are viewing the comet from a different angle (maximum difference of $60^{\circ}$ ), and thus we see a noticeable change in the shape of the lightcurve. Additionally, the peak-to-trough amplitude decreases from $0.50 \pm 0.07 \mathrm{mag}$ to $0.30 \pm 0.1 \mathrm{mag}$ between 2017 and 2018 July (Table 3). Due to the differences in viewing geometry between the two epochs of observations, the change in amplitudes suggests that we are viewing the nucleus at a different aspect ratio. However, additional observations at other viewing geometries would be needed to significantly constrain the pole orientation.

\subsection{Pre-perihelion Lightcurve (2017)}

In order to determine the rotation period of comet $96 \mathrm{P}$, we looked for periodic variations in the lightcurves. As we do not have knowledge of the pole orientation, we only considered the synodic rotation period. However, for most pole orientations, the synodic period should have been within $\sim 2 \mathrm{~s}$ of the sidereal period given that the phase angle bisector angle (Harris et al. 1984) changed by only $\sim 0^{\circ} 3$ between 2017 July 2 and 3 . The observations from 2017 July 2 spanned $\sim 8 \mathrm{hr}$ (see Figure 4) and exhibited a double-peaked lightcurve that repeated after $\sim 4.1 \mathrm{hr}$. This rotation period was further constrained by superimposing the partial lightcurve from the subsequent night, with the data phased to a "trial" period and zero phase set at perihelion (2017 October 27.96). Systematic iterations through the trial period allowed us to make direct "better or worse" comparisons between different rotation periods, as shown in Figure 5. Using this method, we were able to constrain the pre-perihelion rotation period to $4.10 \pm 0.03 \mathrm{hr}$. As the data taken on 2017 July 3 (navy blue circles) do not show signs of a trough nor a peak, the uncertainty in the rotation period is dominated by the uncertainty in the absolute calibration. These uncertainties affected the solution by $\sim 0.02 \mathrm{hr}$, while the uncertainty in the timing for any given absolute calibration was of the order of $\sim 0.01 \mathrm{hr}$. Attempts were also made to constrain the rotation period using the observation from 2017 April 7, but these data were too sparse and the time interval was too long to yield useful constraints, because numerous tightly spaced aliases were possible.

We independently searched for the period using phase dispersion minimization (PDM; Stellingwerf 1978). PDM is a widely used numerical technique for analyzing sparse, nonsinusoidal lightcurves as it does not require uniformly sampled data. The "true" period is indicated by a local minimum in the PDM plot $(\theta)$, as shown in Figure 6 at $4.097 \pm 0.015 \mathrm{hr}$ for the combined 2017 July 2 and 3 data. The uncertainty was quantified by determining the range in the minima of the PDM when the absolute magnitudes on both nights were shifted by their uncertainties in the absolute calibration. This rotation period is in excellent agreement with the rotation period obtained by inspection of the phased lightcurves. We conservatively use the less restrictive period (from phasing) for this epoch.

\subsection{Post-perihelion Lightcurve (2018)}

In the same vein as for the pre-perihelion lightcurves, we phased the post-perihelion data and took an iterative approach to scan through a large number of trial periods. This was done using data from the nights of 2018 July 10/11, July 12, July 31 /August 1, August 1/2, and August 2 as shown in Figure 7. Due to low S/N, the data from 2018 June 24 were not found to be constraining and were thus not used for phasing. We found a 


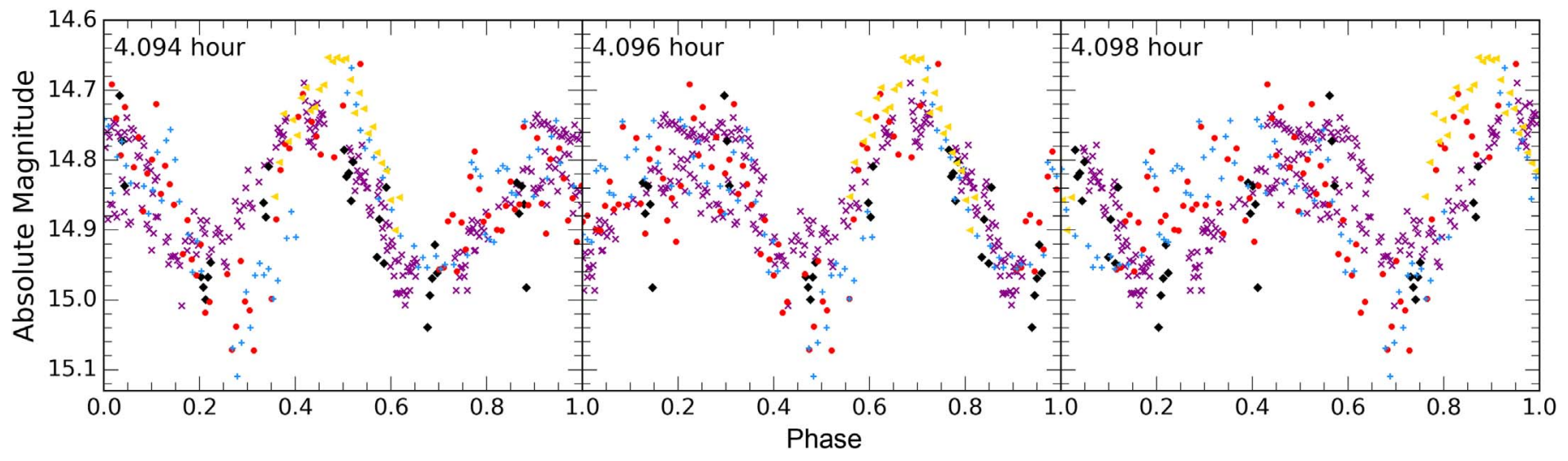

Figure 7. Our reduced pre-perihelion data $\left(H_{r^{\prime}}\right)$ for the nights of 2018 July 10/11, July 12, July 31/August 1, August 1/2, and August 2 phased to 4.094 hr (left), $4.096 \mathrm{hr}$ (middle), and $4.098 \mathrm{hr}$ (right). Colors and symbols are as given in Figure 1. Zero phase was set at the time of perihelion 2017 October 27.96. Data obtained on 2018 July 12 and 2018 July 31 through August 2 have been offset by -0.15 and +0.08 , respectively.

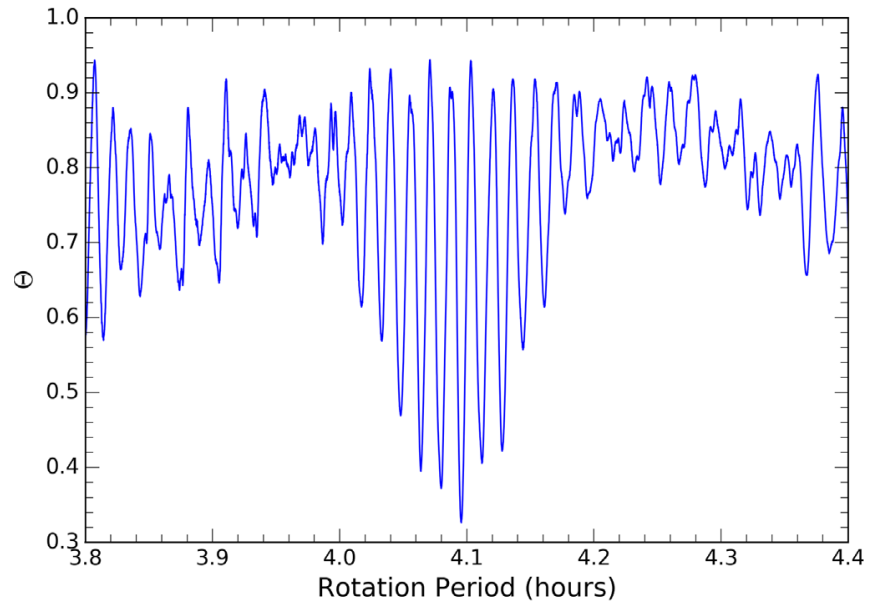

Figure 8. Phase dispersion minimization diagram for the post-perihelion data shown in Figure 7 highlighting a minimum centered on $\sim 4.095 \pm 0.011 \mathrm{hr}$.

best period of $4.096 \pm 0.002 \mathrm{hr}$ through visual phasing of the data.

In order to aid with the comparison and to yield a more robust PDM result, we applied small shifts to the magnitudes of all of the lightcurves in order to bring their extrema into approximate alignment with the lightcurve from 2018 July 10/ 11 , which we found to be the most constraining night. In order to align the data from 2018 July 12, we applied an offset of -0.15 . While this shift is large, it is consistent with the uncertainty in the absolute calibration. The lightcurves from 2018 July 31 /August 1 , August $1 / 2$, and August 2 were all offset by a magnitude of +0.08 mag.

The need for these shifts is likely due to the differences in calibration techniques used on different nights, notably the differences in calibration of the DIA processed images and the traditional photometry images. Further differences could have resulted from using different aperture sizes, which were chosen based on the seeing on a given night. The effect of aperture size on the amplitude can be seen in the August $1 / 2$ lightcurve (light blue plus signs in Figure 7), where the peaks and troughs are not clearly defined despite the maximum and minimum amplitudes being more extreme than on the other nights.

In addition to the misalignment of the peaks and troughs, there is a clear misalignment of the magnitudes at the beginning and end of the night on 2018 July 10 (purple crosses), located at a phase of $\sim 0.4$ in the left panel (Figure 7). While using larger aperture sizes can correct for this misalignment, it also results in significant contamination from field stars, and we have thus elected to not correct for this effect.

The PDM plot for the post-perihelion data (Figure 8) displays a minimum centered on $4.095 \pm 0.011 \mathrm{hr}$. The local minima immediately surrounding $4.095 \mathrm{hr}$ were rejected because some nights were clearly a half-cycle out of phase, while the next local minima moving away from the overall smallest value of $\theta$ clearly showed a misalignment of the data obtained on 2018 July $10 / 11$ and on August $1 / 2$. The alignment of the phased data became significantly worse as the rotation period continued to deviate from $4.096 \mathrm{hr}$, so we are able to eliminate these aliases. The uncertainty in the PDM was assessed using Monte Carlo error analysis in a manner similar to that described in Kokotanekova et al. (2017). This method varies the magnitude of each data point by a random number from a Gaussian distribution with a standard deviation determined by the uncertainty in each point and a mean of zero. This was run 10,000 times, and the standard deviation of all the minima was taken to be the overall uncertainty.

The post-perihelion data obtained in 2018 June-August exhibited significantly lower $\mathrm{S} / \mathrm{N}$ than the 2017 data, due to the worse geometry, that is, the heliocentric and geocentric distances were larger during these later observations. Furthermore, the comet was situated in the midst of the Milky Way and was located within $\sim 90^{\circ}$ from a $\sim 70 \%$ illuminated moon on 2018 June 25. Thus, our observations suffered from significant contamination from a crowded field and scattered light. Even though the scatter in the post-perihelion lightcurves was greater than that of the pre-perihelion data, the larger time baseline allowed us to determine the rotation period to a higher degree of accuracy, yielding a value of $4.096 \pm 0.002 \mathrm{hr}$. Furthermore, despite the noise, we were able to distinguish half-cycle differences, allowing us to avoid aliases. As in 2017, the viewing geometry changed very little between our observations, with the phase angle bisector differing by only $\sim 3^{\circ}$ from 2018 June 25 to August 2, and yielding likely synodic and sidereal periods in agreement to within $\sim 1 \mathrm{~s}$.

\section{Discussion}

Our observations throughout the 2017/2018 apparition suggest little or no change in the rotation period during this passage, with a change of $<0.036 \mathrm{hr}$ (130 s, or 2.2 minutes). At 
first glance, this lack of change is not surprising based on the large size of the nucleus, $3.41 \pm 0.24 \mathrm{~km}$, and the low active fraction of the comet (e.g., Combi et al. 2011). Similar results have been found for other large, relatively inactive nuclei, such as 10P/Tempel 2 (Knight et al. 2011), 49P/Arend-Rigaux (Eisner et al. 2017), and 14P/Wolf, 143P/Kowal-Mrkos, and 162P/Siding Spring (Kokotanekova et al. 2018).

However, $96 \mathrm{P}$ is very active near perihelion, with $Q_{\mathrm{H} 2 \mathrm{O}} \sim 4 \times 10^{29}$ molecules $\mathrm{s}^{-1}$ (Combi et al. 2019). We would expect this activity to exert a significant torque on the nucleus and thus potentially result in a measurable change in rotation period. Furthermore, Mueller \& Samarasinha (2018) argued that $96 \mathrm{P}$ is likely to exhibit a large change in rotation period based on models that relate changes in rotation period of Jupiter-family comets to other physical parameters. They predict the maximum change in rotation period of comet $96 \mathrm{P}$ to be 9.1 minutes per apparition based on previous estimates for nucleus size and rotation period. Updating their calculation with our new rotation period and nuclear radius lowers this to 2.5 minutes, although for their less extreme torque cases, the predicted change in rotation period is less than our upper limit.

In order to examine this further, we compared the rotation period of 96P during the 2017 apparition to previous results found in the literature. To the best of our knowledge, the only other time that attempts were made to quantify the rotation period was during the 1995/1996 apparition, where Meech (1996) reported a period of $6.38 \mathrm{hr}$. This is notably longer than the periods determined for the most recent apparition $(4.10 \pm 0.03 \mathrm{hr}$ and $4.096 \pm 0.002 \mathrm{hr}$ before and after perihelion, respectively), possibly suggesting a significant change in rotation period over the course of the $21 \mathrm{yr}$ between the two measurements. If we assume that the rotation period decreased by 9.1 minutes with every apparition since 1996, as predicted by Mueller \& Samarasinha (2018), we obtain a total change of 36.4 minutes prior to the 2017 perihelion passage, bringing the period to $\sim 5.77 \mathrm{hr}$. This value remains significantly different from our determined value for the 2017 apparition. As Meech's (1996) data are not shown, one possible explanation is that an alias near $4.1 \mathrm{hr}$ exists. Notably, if their period was a 3:2 alias, it would imply a period of $\sim 4.25 \mathrm{hr}$. To get to our period of $4.1 \mathrm{hr}$ would require a much more reasonable change of $\sim 2.2$ minutes per orbit, which is within our uncertainty and therefore not necessarily detectable.

Alternatively, and less likely in our opinion, the rotation period of comet 96P could have been drastically changed by processes that are not considered by the model presented by Mueller \& Samarasinha (2018). Certain processes, for example, could be more prominent, due to the unusually small perihelion distance that comet $96 \mathrm{P}$ reaches. At perihelion, equilibrium temperatures reach $\sim 800 \mathrm{~K}$ and subsolar temperatures can exceed $1000 \mathrm{~K}$, potentially resulting in the sublimation of refractory materials (e.g., Mann et al. 2004).

A further possibility is that the rotation period has changed as a result of fragmentation. Comet $96 \mathrm{P}$ is known to fragment, with dynamical evidence that it is the parent of at least two groups of near-Sun comets (Ohtsuka et al. 2003; Sekanina \& Chodas 2005). Additionally, two and three fragments were observed with $\mathrm{SOHO}$ during the 2012 and 2017 apparitions, respectively. The process of fragmentation can cause changes to the moment of inertia of the nucleus, possibly leading to a change in the rotation period (e.g., Samarasinha et al. 2004). Even though fragmentation may result in a change in rotation period, the effect is likely small in the case of comet 96P because the fragments are apparently dwarfed by the main body (based on their apparent brightnesses in SOHO images). Nonetheless, such an event would also lead to more exposed surface ices. Vigorous activity from these newly exposed ices might result in a torque that acts to change the rotation period far more efficiently than the fragmentation itself. We think that this latter explanation is unlikely as freshly exposed ice should result in increased gas production, which was not seen by Combi et al. (2019), who report similar SOHO-SWAN water production rates for the 1996-2012 apparitions.

Water production rates were further used to investigate the lack of an observable coma. In Section 3.1 we showed that the contribution from the coma is negligible and thus that we can assume that we are observing a bare nucleus. This is somewhat surprising based on extrapolation of the water production rates determined by Combi et al. (2011). They found that the water production rate varies as $2.5 \times 10^{27} r_{\mathrm{H}}^{-2.5}$ before perihelion and as $4.6 \times 10^{27} r_{\mathrm{H}}^{-1.9}$ after perihelion in units of molecules per second. Assuming that this relationship holds beyond the distances observed by $S O H O-S W A N$ (out to $r_{\mathrm{H}} \sim 0.8 \mathrm{au}$ ), and using Schleicher (2008)'s dust-to-gas ratio measurements, we would expect the coma flux to be at least as bright as the nucleus flux during our smallest $r_{\mathrm{H}}$ observations at $2.270 \mathrm{au}$ on 2017 July 2. The "total" magnitudes reported to the Minor Planet Center (MPC) for 96P are consistent with our findings that $96 \mathrm{P}$ had minimal coma. An unweighted fit to all total magnitudes in the MPC database at $r_{\mathrm{H}}<2 \mathrm{au}$, normalized to $r_{\mathrm{H}}=\Delta=1$ au and with a linear phase angle correction of 0.04 mag per degree, suggests the brightness varies proportional to $r_{\mathrm{H}}^{-4.5}$, and the "total" magnitudes converge with the "nucleus" magnitudes around 2 au. Although sparse, reported magnitudes at larger heliocentric distances are suggestive of no coma. We caution overinterpreting such aggregated MPC data because a variety of techniques, filters, and aperture sizes are used; however, they support our conclusion that $96 \mathrm{P}$ is essentially inactive by $2.3 \mathrm{au}$. The lack of detectable coma could be due to a strong seasonal effect, that is, the active regions are located such that they turn on/off rapidly somewhere between 0.8 and $2.3 \mathrm{au}$. Alternatively it could be due to the comet possessing an even lower dust-to-gas ratio at 2.3 au than found by Schleicher (2008) at 1.8 au (which was already low relative to other comets in the A'Hearn et al. 1995 database); for example, we do not see dust, but there is still gas that we do not detect.

Based on the assumption that we are observing a bare nucleus, we investigated the color of $96 \mathrm{P}$ during the most recent apparition. While the color itself does not reveal much in terms of the physical properties of the nucleus, the direct comparison of this value to that of other bodies in the solar system can disclose information on the origin or evolution of the comet. Our results revealed that the nucleus of comet $96 \mathrm{P}$ is significantly more blue than the average nuclei of Jupiterfamily comets. This could, once again, be a result of the intense heat that the comet endures during its close approach to the Sun, and could be indicative of a more evolved comet surface. This argument is strengthened by the similarity in color to $322 \mathrm{P}$, which has $q=0.05$ au but whose cometary origin is uncertain (Knight et al. 2016).

The color of $96 \mathrm{P}$ is also notably similar to that of the two Halley-type comets that have published nuclear colors, comet $1 \mathrm{P} /$ Halley and C/2001 OG108. Although it is dangerous to draw conclusions from a sample size of two comets, at face 
value the likenesses in all three color metrics could indicate that $96 \mathrm{P}$ is a Halley-type comet (recall that 96P's peculiar resonance with Jupiter precludes making a firm determination of its origin) and that this color is a signature of Halley-type comets or Oort cloud objects. Alternatively, the color could be an evolutionary trait, with comets becoming less red over time. Compared to Kuiper Belt Objects/Centaurs, comets appear to lose more ultrared matter when they become active, possibly due to resurfacing (Jewitt 2018). This, however, may not apply to comets that reach extremely close perihelion distances because they have less fallback of material.

Comet 96P's nuclear properties were further compared to those of other comets. As already discussed, the nucleus of comet $96 \mathrm{P}$ rotates with a period of $\sim 4.1 \mathrm{hr}$, placing it among the fastest-rotating comet nuclei known (Kokotanekova et al. 2017). Combined with the axial ratio of at least $\sim 1.5: 1$, this suggests that either its density is unusually high (e.g., $>0.6 \mathrm{~g} \mathrm{~cm}^{-3}$ for a strengthless body) or that it has more internal strength than is typically attributed to comet nuclei. The only unquestionably cometary object thought to have a significantly more extreme combination of rotation period and axial ratio is Component $\mathrm{C}$ of $73 \mathrm{P} /$ Schwassmann-Wachmann 3 , whose rotation period was measured after it underwent rampant fragmentation (Drahus et al. 2010). However, other observations (Nolan et al. 2006; Dykhuis et al. 2012) report rotation periods for $73 \mathrm{P}-\mathrm{C}$ of at least $10 \mathrm{hr}$, and $96 \mathrm{P}$ may, in fact, be the most extreme rotator known. Thus, it seems likely that $96 \mathrm{P}$ may currently be at, or near, its rotational spin limit. We speculate that rotationally driven fragmentation may be a common, or even dominant, source of the dynamically related objects that appear to have split from 96P both recently (e.g., the fragments seen by $\mathrm{SOHO}$ in 2012 and 2017) and centuries ago (e.g., the "Machholz Complex," see Ohtsuka et al. 2003; Sekanina \& Chodas 2005).

Two other potentially cometary objects stand out as having extreme combinations of rotation period and axial ratio: comet 322P/SOHO 1 (Knight et al. 2016) and interstellar object 1I/ 'Oumuamua (e.g., Meech et al. 2017; Drahus et al. 2018; Fraser et al. 2018). Intriguingly, both objects have $V-R$ and $B-V$ colors similar to $96 \mathrm{P}$. Although 'Oumuamua was initially thought to be asteroidal based on the lack of detectable coma (e.g., Jewitt et al. 2017; Knight et al. 2017; Meech et al. 2017; Bolin et al. 2018), later astrometric analysis by Micheli et al. (2018) revealed unequivocal nongravitational acceleration that the authors attributed to cometary outgassing. Micheli et al. concluded that such activity could have gone undetected if 'Oumuamua was depleted in $\mathrm{CN}$ relative to water by a factor of at least 15 and had large dust grains not detectable at visible wavelengths. As previously noted, $96 \mathrm{P}$ was suggested to have potentially been of interstellar origin based on its severe $\mathrm{CN}$ depletion relative to water (by a factor of 72; Schleicher 2008), and it has a low dust-to-gas ratio as derived from visible observations. Thus, the additional similarities between the nucleus of 96P and 'Oumuamua might strengthen the argument for $96 \mathrm{P}$ having had an interstellar origin (and that these surface properties were acquired during millions of years of cosmic-ray bombardment between systems, rather than having been due to evolution in our solar system, e.g., Fitzsimmons et al. 2018). Another possible interpretation is that, as speculated by $\mathrm{Ye}$ et al. (2017) and Ćuk (2018), 'Oumuamua may have been ejected from its natal system during a close passage to its host star (or stars if a binary). In that scenario, the properties observed as it passed through our solar system could have been caused by evolutionary effects that are due to repeated close approaches to its original parent star, suggesting that 96P's unusual properties are evolutionary rather than indicative of the environment in which it formed. As exciting as such speculation is, the similarities between 96P and 'Oumuamua could simply be coincidental, because the $R-I$ colors, though poorly constrained, are quite different and may be diagnostic of compositional differences that are due to the presence of silicate absorption at $\sim 1$ micron (e.g., Reddy et al. 2015 and references therein)

As noted in the introduction, 322P was a motivator of the current investigation because of its combination of very small perihelion distance $(0.054 \mathrm{au})$, unusual colors, and fast rotation period. The similarity of $96 \mathrm{P}$ to $322 \mathrm{P}$ (and as just discussed, possibly 'Oumuamua) in all three aspects suggests that there may be a trend with perihelion distance, perhaps due to surface properties changing as a result of the intense heating near perihelion. In particular, annealing of the upper layers may give a comet strength, allowing it to sustain a spin rate above the nominal rotational breakup limit. Knight et al. (2016) showed that $322 \mathrm{P}$ had a high albedo (0.09-0.42); if this, too, is a result of the small perihelion distance, then $96 \mathrm{P}$ may be substantially smaller than has otherwise been assumed. A smaller size would also mean its active fraction is substantially larger than estimated by Combi et al. (2011), implying it is much less evolved. Further investigation of 96P's nucleus properties in order to investigate evolutionary trends with perihelion distance would be highly desirable since $322 \mathrm{P}$ and $96 \mathrm{P}$ are the only periodic comets observed by traditional (i.e., non-coronagraphic) telescopes with perihelion distances smaller than 2P/Encke's 0.336 au.

\section{Conclusion}

We observed comet $96 \mathrm{P} / \mathrm{Machholz} 1$ on a total of nine nights both pre- and post-perihelion during its 2017/2018 apparition. Throughout our observations, it did not exhibit any evidence of a coma, a somewhat surprising result given that we observed at heliocentric distances as low as $2.3 \mathrm{au}$, and suggesting that $96 \mathrm{P}$ has either strong seasonal activity or that its upper layers are depleted in volatiles by repeated close perihelion passages. We did not see any evidence of the fragments seen accompanying $96 \mathrm{P}$ in the $\mathrm{SOHO}$ fields of view around perihelion in 2012 and 2017. Our best upper limit for such fragments is a radius of $\sim 100 \mathrm{~m}$, though the relatively small field of view likely does not meaningfully constrain their time of formation.

We obtained complete rotational lightcurves both pre- and post-perihelion, finding a double-peaked lightcurve with one strong peak and one wide, flat peak, and two clean minima. We measured rotation periods of $4.10 \pm 0.03 \mathrm{hr}$ and $4.096 \pm$ $0.002 \mathrm{hr}$ before and after perihelion, respectively. Thus, to within the uncertainties, the rotation period did not change during the apparition. Based on the assumption that we observed the bare nucleus, we estimated the effective radius to be $3.41 \pm 0.24 \mathrm{~km}$ and the minimal axial ratio to be $1.6+/-0.1: 1$. This is the third fastest rotation period ever measured for a comet nucleus and, along with the axial ratio, suggests that either its density is unusually high or that $96 \mathrm{P}$ has more internal strength than typical comet nuclei. The comet may be at or near its rotational spin limit, suggesting a possible 
mechanism for the shedding of the small fragments seen near perihelion.

We also measured nucleus colors both pre- and postperihelion, finding average values of $g^{\prime}-r^{\prime}=0.50 \pm 0.04$, $r^{\prime}-i^{\prime}=0.17 \pm 0.03$, and $i^{\prime}-z^{\prime}=0.06 \pm 0.04$. These colors are bluer than typical Jupiter-family comet nuclei, but similar to the two published Halley-type comet nuclei, potentially indicating that $96 \mathrm{P}$ evolved from a Halley-type orbit. If such a link could be firmly established, it might indicate that blue nucleus colors are an intrinsic characteristic of such orbits. Similar blue colors have also been observed for near-Sun comet 322P/SOHO and for interstellar object 1I/'Oumuamua, suggesting a possible evolutionary cause, due to either a small perihelion distance or (less likely) space weathering during an interstellar journey.

The high inclination of 96P causes it to be very far south during most of its orbit, limiting the telescopes with which it can be effectively studied. Its next apparition in 2023 will be especially poor, but the 2028 apparition will be its best since discovery, when it passes just 0.32 au from Earth prior to perihelion. We encourage further investigations of this beguiling object, and we especially advocate the use of the James Webb Space Telescope for thermal infrared studies to constrain the size, albedo, and grain size distribution.

We thank the referee for a thorough and helpful review and Carlos Corco, Patricio Ugarte, Jacqueline Seron, Juan Espinoza, Alfredo Zenteno, Regis Cartier, Cesar Briceno, and Sean Points for help obtaining our SOAR observations. N.E., M.M.K., and M.S.P.K. were supported by NASA Near Earth Object Observations grant No. NNX17AK15G. This research made use of Astropy, a community-developed core Python package for Astronomy (Astropy Collaboration et al. 2013), as well as PyAstronomy. This work also used SAOIMAGE DS9, developed by Smithsonian Astrophysical Observatory and the "Aladin sky atlas" developed at Centre de Données astronomiques de Strasbourg (CDS), Strasbourg Observatory, France (Bonnarel et al. 2000). Additionally, GMOS standard starfinding charts prepared by the AAO ITSO Office in conjunction with Macquarie University's PACE (Professional and Community Engagement) program were used.

The Pan-STARRS1 Surveys (PS1) and the PS1 public science archive have been made possible through contributions by the Institute for Astronomy, the University of Hawaii, the Pan-STARRS Project Office, the Max-Planck Society and its participating institutes, the Max Planck Institute for Astronomy, Heidelberg, and the Max Planck Institute for Extraterrestrial Physics, Garching, The Johns Hopkins University, Durham University, the University of Edinburgh, the Queen's University Belfast, the Harvard-Smithsonian Center for Astrophysics, the Las Cumbres Observatory Global Telescope Network Incorporated, the National Central University of Taiwan, the Space Telescope Science Institute, the National Aeronautics and Space Administration under grant No. NNX08AR22G issued through the Planetary Science Division of the NASA Science Mission Directorate, the National Science Foundation grant No. AST-1238877, the University of Maryland, Eotvos Lorand University (ELTE), the Los Alamos National Laboratory, and the Gordon and Betty Moore Foundation.

Facilities: SOAR (Goodman, SOI), William Herschel Telescope (ACAM), New Technology Telescope (EFOSC2).
Software: Aladin Sky Atlas, Astropy (Astropy Collaboration et al. 2013), IDL, IRAF, SAOImage DS9.

\section{ORCID iDs}

M. M. Knight (iD https://orcid.org/0000-0003-2781-6897

C. Snodgrass (ii) https://orcid.org/0000-0001-9328-2905

M. S. P. Kelley (i) https://orcid.org/0000-0002-6702-7676

A. Fitzsimmons (iD https://orcid.org/0000-0003-0250-9911

R. Kokotanekova (iD https://orcid.org/0000-0003-4617-8878

\section{References}

Astropy Collaboration, Robitaille, T. P., Tollerud, E. J., et al. 2013, A\&A, 558, A33

A’Hearn, M. F., Millis, R. L., Schleicher, D. G., Osip, D. J., \& Birch, P. V. 1995, Icar, 118, 223

Bailey, M. E., Chambers, J. E., \& Hahn, G. 1992, A\&A, 257, 315

Bannister, M. T., Schwamb, M. E., Fraser, W. C., et al. 2017, ApJL, 851, L38

Battams, K., \& Knight, M. M. 2017, RSPTA, 375, 20160257

Battams, K., \& Lui, L. 2013, CBET, 3631

Benn, C., Dee, K., \& Agócs, T. 2008, Proc., SPIE, 7014, 70146X

Bolin, B. T., Weaver, H. A., Fernandez, Y. R., et al. 2018, ApJL, 852, L2

Bonnarel, F., Ziaeepour, H., Bartlett, J. G., et al. 2000, A\&AS, 143, 33

Bramich, D. M. 2008, MNRAS, 386, L77

Bramich, D. M., Horne, K., Albrow, M. D., et al. 2013, MNRAS, 428, 2275

Buzzoni, B., Delabre, B., Dekker, H., et al. 1984, Msngr, 38, 9

Carusi, A., Kresak, L., Perozzi, E., \& Vasecchi, G. B. 1987, A\&A, 187, 899

Chambers, K. C. 2017, yCat, 2349

Clemens, J. C., Crain, J. A., \& Anderson, R. 2004, Proc. SPIE, 5492, 331

Colina, L., Bohlin, R. C., \& Castelli, F. 1996, AJ, 112, 307

Combi, M. R., Boyd, Z., Lee, Y., et al. 2011, Icar, 216, 449

Combi, M. R., Makinen, T. T., Bertaux, J.-L., Quemerais, E., \& Ferron, S. 2019, Icar, 317, 610

Ćuk, M. 2018, ApJL, 852, L15

Drahus, M., Guzik, P., Waniak, W., et al. 2018, NatAs, 2, 407

Drahus, M., Kueppers, M., Jarchow, C., et al. 2010, A\&A, 510, A55

Dykhuis, M. J., Samarasinha, N. H., Mueller, B. E. A., \& Storm, S. P. 2012, AAS DPS Meeting, 44, 314.10

Eisner, N., Knight, M. M., Battams, K., Kelley, M. S., \& Snodgrass, C. 2018, COSPAR, B0.3-0017-18

Eisner, N., Knight, M. M., \& Schleicher, D. G. 2017, AJ, 154, 196

Fitzsimmons, A., Snodgrass, C., Rozitis, B., et al. 2018, NatAs, 2, 133

Fraser, W. C., Pravec, P., Fitzsimmons, A., et al. 2018, NatAs, 2, 383

Fukugita, M., Ichikawa, T., Gunn, J. E., et al. 1996, AJ, 111, 1748

Granvik, M., Morbidelli, A., Jedicke, R., et al. 2016, Natur, 530, 303

Green, D. W. E., Rickman, H., Porter, A. C., \& Meech, K. J. 1990, Sci, 247, 1063

Grynko, Y., Jockers, K., \& Schwenn, R. 2004, A\&A, 427, 755

Hainaut, O. R., Boehnhardt, H., \& Protopapa, S. 2012, A\&A, 546, A115

Harris, A. W., Young, J. W., Scaltriti, F., \& Zappala, V. 1984, Icar, 57, 251

Jewitt, D. 1991, in IAU Coll. 116, Comets in the Post-Halley Era, ed. R. L. Newburn, M. Neugebauer, \& J. Rahe (Dordrecht: Kluwer) Jewitt, D. 2018, arXiv:1808.04885

Jewitt, D., Luu, J., Rajagopal, J., et al. 2017, ApJL, 850, L36

Jones, G. H., Knight, M. M., Battams, K., et al. 2018, SSRv, 214, 20

Knight, M. M. 2008, PhD thesis, Univ. Maryland, College Park

Knight, M. M., Farnham, T. L., Schleicher, D. G., \& Schwieterman, E. W. 2011, AJ, 141, 2

Knight, M. M., Fitzsimmons, A., Kelley, M. S. P., \& Snodgrass, C. 2016, ApJL, 823, L6

Knight, M. M., Protopapa, S., Kelley, M. S. P., et al. 2017, ApJL, 851, L31

Knight, M. M., Schleicher, D. G., Farnham, T. L., Schwieterman, E. W., \& Christensen, S. R. 2012, AJ, 144, 153

Knight, M. M., \& Walsh, K. J. 2013, ApJL, 776, L5

Kokotanekova, R., Snodgrass, C., Lacerda, P., et al. 2017, MNRAS, 471, 2974 Kokotanekova, R., Snodgrass, C., Lacerda, P., et al. 2018, MNRAS, 479, 4665

Lamy, P., Faury, G., Llebaria, A., et al. 2013, Icar, 226, 1350

Lamy, P., \& Toth, I. 2009, Icar, 201, 674

Lamy, P. L., Toth, I., Fernandez, Y. R., \& Weaver, H. A. 2004, in Comets II, ed. M. C. Festou, H. U. Keller, \& H. A. Weaver (Tucson, AZ: Univ. Arizona Press), 223 
Lamy, P. L., Toth, I., Weaver, H. A., A'Hearn, M. F., \& Jorda, L. 2009, A\&A, 508,1045

Langland-Shula, L. E., \& Smith, G. H. 2007, ApJL, 664, L119

Levison, H. F., \& Dones, L. 2014, in Encyclopedia of the Solar System, ed. T. Spohn, D. Breuer, \& T. V. Johnson (3rd ed.; Amsterdam: Elsevier), 708

Licandro, J., Tancredi, G., Lindgren, M., Rickman, H., \& Hutton, R. G. 2000, Icar, 147,161

Lowry, S., Duddy, S. R., Rozitis, B., et al. 2012, A\&A, 548, A12

Lupton, R. 2005, Transformations between SDSS Magnitudes and $\mathrm{UBVR}_{\mathrm{c}} \mathrm{I}_{\mathrm{c}}$, http://www.sdss.org/dr5/algorithms/sdssUBVRITransform.html

Mann, I., Kimura, H., Biesecker, D. A., et al. 2004, SSRv, 110, 269

Marsden, B. G. 2005, ARA\&A, 43, 75

McIntosh, B. A. 1990, Icar, 86, 299

Meech, K. 1996, Asteroids, Comets, and Meteors 1996 Meeting, http://www. ifa.hawaii.edu/ meech/papers/acm96.pdf

Meech, K. J., Hainaut, O. R., \& Marsden, B. G. 2004, Icar, 170, 463

Meech, K. J., Weryk, R., Micheli, M., et al. 2017, Natur, 552, 378

Micheli, M., Farnocchia, D., Meech, K. J., et al. 2018, Natur, 559, 223

Mueller, B. E. A., \& Samarasinha, N. 2018, AJ, 156, 107

Nolan, M. C., Harmon, J. K., Howell, E. S., et al. 2006, BAAS, 38, 504

Ohtsuka, K., Nakano, S., \& Yoshikawa, M. 2003, PASJ, 55, 321
Reddy, V., Dunn, T. L., Thomas, C. A., Moskovitz, N. A., \& Burbine, T. H. 2015, in Asteroids IV, ed. P. Michel, F. E. DeMeo, \& W. F. Bottke (Tucson, AZ: Univ. Arizona Press), 43

Russell, H. N. 1916, ApJ, 43, 173

Samarasinha, N. H., Mueller, B. E. A., Belton, M. J. S., \& Jorda, L. 2004, in Comets II, ed. M. C. Festou, H. U. Keller, \& H. A. Weaver (Tucson, AZ: Univ. Arizona Press), 281

Schleicher, D. G. 2008, AJ, 136, 2204

Sekanina, Z. 1988, AJ, 96, 1455

Sekanina, Z. 1990, AJ, 99, 1268

Sekanina, Z. 1991, JRASC, 85, 324

Sekanina, Z. 2013, CBET, 3642

Sekanina, Z., \& Chodas, P. W. 2005, ApJSS, 161, 551

Smith, J. A., Tucker, D. L., Kent, S., et al. 2002, AJ, 123, 2121

Snodgrass, C., Fitzsimmons, A., \& Lowry, S. C. 2005, A\&A, 444, 287

Snodgrass, C., Jehin, E., Manfoid, J., et al. 2016, A\&A, 588, A80

Snodgrass, C., Tubiana, C., Bramich, D. M., et al. 2013, A\&A, 557, A33

Stellingwerf, R. F. 1978, ApJ, 224, 953

Tonry, J. L., Stubbs, C. W., \& Lykke, K. R. 2012, ApJ, 750, 99

Walker, A. R., Boccas, M., Bonati, M., et al. 2003, Proc. SPIE, 4841, 286

Ye, Q. Z., Zhang, Q., Kelley, M. S., \& Brown, P. G. 2017, ApJL, 851, L5 